\title{
Assessment of Computational Fluid Dynamic for Surface Combatant 5415 at Straight Ahead and Static Drift $\beta=20$ deg
}

S. Bhushan, Department of Mechanical Engineering, Mississippi State University, Starkville, MS 39759, e-mail: bhushan@me.msstate.edu

H. Yoon, IIHR Hydroscience and Engineering, University of Iowa, Iowa City, IA 52246, e-mail: hyun-se-yoon@uiowa.edu

F. Stern, IIHR Hydroscience and Engineering, University of Iowa, Iowa City, IA 52246, e-mail: frederick-stern@uiowa.edu

E. Guilmineau, LHEEA—UMR6598 CNRS, Centrale Nantes, Nantes 44321, France, e-mail: Emmanuel.Guilmineau@ec-nantes.fr

M. Visonneau, LHEEA_UMR6598 CNRS, Centrale Nantes, Nantes 44321, France, e-mail: Michel.Visonneau@ec-nantes.fr

S. L. Toxopeus, Maritime Research Institute Netherlands, 6708 PM, Wageningen, The Netherlands, e-mail: S.L.Toxopeus@marin.nl

C. Simonsen, FORCE Technology, DK-2605 Brøndby, Denmark, e-mail: cds@force.dk

S. Aram, Naval Surface Warfare Center, Carderock Division, West Bethesda, MD 20817, e-mail: shawn.aram@navy.mil

S-E Kim, Naval Surface Warfare Center, Carderock Division, West Bethesda, MD 20817, e-mail: sungeun.kim@navy.mil

G. Grigoropoulos, National Technical University of Athens, Zografou 15780, Greece, e-mail: gregory@central.ntua.gr

Collaboration is described on assessment of computational fluid dynamics (CFD) predic-tions for surface combatant model 5415 at static drift b $1 / 40$ deg and 20 deg using recent tomographic particle image velocimetry (TPIV) experiments. Assessment includes Nversion verification and validation to determine the confidence intervals for CFD solu-tions/codes, and vortex onset, progression, instability, and turbulent kinetic energy (TKE) budget analysis. The increase in b shows the following trends. Forces and moment increase quadratically/cubically, and become unsteady due to shear layer, Karman and flapping instabilities on the bow. Wave elevation becomes asymmetric; its amplitude increases, but the total wave elevation angle remains same. The vortex strength and TKE increase by about two orders of magnitude, and for large $b$, the primary vortices exhibit helical mode instability similar to those for delta wings. Forces and moment for both $\mathrm{b}$ and wave elevation for $\mathrm{b} 1 / 40$ deg are compared within $4 \%$ of the data, and are validated at $7 \%$ interval. Wave elevation for $\mathrm{b} 1 / 420 \mathrm{deg}$, and vortex core location and velocities for both $\mathrm{b}$ are compared within $9 \%$ of the data, and are validated at $12 \%$ interval. The vortex strength and TKE predictions show large $70 \%$ errors and equally large scatter and are not validated. Thus, both errors and scatter need reduction. TKE budgets show transport of turbulence into the separation bubble similar to canonical cases, but pressure trans-port is dominant for ship flows. Improved CFD predictions require better grids and/or turbulence models. Investigations of solution-adaptive mesh refinement for better grid design and hybrid Reynolds-averaged Navier-Stokes/large eddy simulation models for improved turbulent flow predictions are highest priority.

\section{Introduction}

Prediction of three-dimensional (3D) separation including onset and progression of vortices is a grand challenge for ship hydrodynamics due to relatively bluff hull forms with attendant thick boundary layer and wake; appendages such as bow bulb/ sonar dome and shafts/struts; free-surface/Froude number (Fr) effects; and high Reynolds numbers (Re). Hull generated vortices can significantly affect the propeller inflow, thereby adversely affecting the ship performance [1]. Thus, an improved understanding of the onset and progression of vortices is required for improved hull, appendage, and propulsor design. The following provides a brief overview of the recent studies using large grids and advanced turbulence models for static drift simulations 
focusing on assessment of computational fluid dynamics (CFD) predictive capability.

The Gothenburg 2010 Ship Hydrodynamics workshop (G2010) assessed CFD predictions for tanker (KVLCC2), container (KCS), and surface combatant (5415) hull forms for calm water resistance, sinkage, and trim among other conditions [2]. The results included steady Reynolds-averaged Navier-Stokes (RANS), unsteady RANS (URANS) and detached eddy simulation (DES) with isotropic and anisotropic turbulence models on grid sizes varying from $<1$ million (M) to $300 \mathrm{M}$ cells. Anisotropic turbulence models showed improvement over isotropic models for the prediction of turbulence structures. However, URANS was overly diffused/dissipative even for grids with $>10 \mathrm{M}$ points and fourth-order convection schemes. Large grid DES provided detailed flow topology, and for the first time helped identify that the closed streamlines at 5415 mid-girth observed in the experiments were the sonar dome (SDV) and forebody keel (FBKV) vortices. However, showed modeled stress depletion and grid induced separation in the boundary layer for bluff bodies, and under resolved turbulence for slender bodies. The study did not include simulations on systematic refined grids. Thus, it was impossible to determine the relative contributions of the grid and turbulence modeling errors. In addition, the available experimental data were too sparse and lacked turbulence measurements for detailed validation.

The SIMMAN 2008 workshop assessed system-based methods and RANS/URANS predictions on up to $7 \mathrm{M}$ cells for static and dynamic maneuvers in calm water [3]. RANS/URANS predictions were in good agreement with the experiments for the forces and moment for small amplitude maneuvers, and local flow for pure sway and yaw at moderate rates. The study concluded that coarse grids and turbulence model deficiencies attributed to the large errors for large amplitude maneuvers.

Xing et al. [4] performed URANS and DES for KVLCC2 at drift angles $\beta=0 \mathrm{deg}, 12 \mathrm{deg}$, and $30 \mathrm{deg}$, including detailed turbulent kinetic energy (TKE) budget and instability analysis. The predictions for $\beta=30 \mathrm{deg}$ showed spiraling vortices with helical mode instability similar to the delta wing vortices, which could not be validated due to lack of experimental data.

Visonneau et al. [5] performed URANS and DES for the Japan bulk carrier, which replaced KVLCC2 as the tanker test case at the Tokyo 2015 CFD Workshop [6], as a post workshop contribution. Simulations used grids with up to $66 \mathrm{M}$ cells for the validation of integral and local flow variables, including the performance of an energy saving device on propulsive efficiency. URANS predicted the mean axial velocity along the vortex core well, but significantly underpredicted the TKE. DES showed better results for both axial velocity and TKE. The study also identified that detailed validations could not be performed due to lack of experimental data.

The sea facet of the NATO AVT 183 working group on Reliable Prediction of Separated Flow Onset and Progression for Air and Sea Vehicles focused on addressing the limitations of the previous studies using new experimental validation data for KVLCC2, Delft Catamaran (DC) and 5415 test cases. Study included 33 contributions from nine institutes and ten countries using in-house and commercial codes for assessment of CFD prediction capability [7].

This study focuses on the assessment of CFD prediction capability for separated flow onset and progression induced by 5415 at $\beta=0 \mathrm{deg}$ and $20 \mathrm{deg}$ using forces and moment, wave elevation, and mean and turbulent flow in the vortex core as the validation variables and adaptive grid verification studies. The international collaboration for the 5415 test cases involved seven institutions from five countries using five different codes with different turbulence and free-surface modeling and numerical methods. Towing tank tomographic particle image velocimetry (TPIV) experiments enabled detailed analysis of the physics [8], and the integrated experiments and simulations provide physical explanations for the separated flow onset and progression. Large adaptive grids were used with about $45 \mathrm{M}$ and $70 \mathrm{M}$ cells for $\beta=0 \mathrm{deg}$ and $20 \mathrm{deg}$, respectively. For $\beta=0$, deg grid sizes are sufficiently large compared to G2010, which helps to evaluate deficiencies due to turbulence model and grid sizes. Solution verification and validation $(\mathrm{V} \& \mathrm{~V})$ follows Stern et al. [9]. Verification uses either factor of safety [10] or least square [11] methods. In addition, a recently developed statistical approach for CFD state-of-the-art (SoA) assessment- $\mathrm{N}$-version $\mathrm{V} \& \mathrm{~V}$ [12], is used for assessments for multiple code contributions. The $\mathrm{N}$-version verification focuses on the scatter in the solutions, whereas $\mathrm{N}$-version validation focuses on the scatter in the absolute errors. ${ }^{2}$ The experiments provided global and local measurements for CFD validation; and once validated, CFD fills in the sparse experimental data enabling detailed diagnostics. Analysis of vortex core and TKE budgets help understand vortex separation and instability mechanism and explain the vortex onset and progression physics. Discussions also focus on wave pattern, breaking, and induced vortices. The paper provides an overview of the results, and readers should refer to Bhushan et al. [13] for detailed results.

\section{Experimental Data, Computational Fluid Dynamics Codes and Setup, Assessment Methods, and Solution Verification}

2.1 Experimental Validation Variables and Uncertainties. The experiments were conducted in a $3 \mathrm{~m} \times 3 \mathrm{~m} \times 100 \mathrm{~m}$ towing tank using an $L=3.048 \mathrm{~m}$ long scaled model of 5415 . The model was unappended except for the port and starboard bilge keels, i.e., not equipped with propellers, shafts, struts, or rudders. Figures $1(a)$ and $1(b)$ show the hull form and the ship-fixed coordinate system. The $x$-, $y$-, $z$-axes are in the streamwise, spanwise, and normal to the calm water surface (positive upward) directions, respectively, with the origin located at the forward perpendicular center plane. The experiments were performed for straight-ahead $\beta=0$ deg and two static drift angles $\beta=10 \mathrm{deg}$ and $20 \mathrm{deg}$. For all the tests, the model was towed at the center of gravity $(x=0.504$, $y=0$ and $z=0.05315)$ with carriage speed $U_{C}=1.531 \mathrm{~m} / \mathrm{s}$ corresponding to $\mathrm{Fr}=0.28$ and $\mathrm{Re}=4.65 \times 10^{6}$ based on water temperature of $20^{\circ} \mathrm{C}$. The model was sunken and trimmed to the fixed sinkage $\left(\sigma=1.92 \times 10^{-3}\right)$ and trim $(\tau=-0.136 \mathrm{deg}$, positive bow up) corresponding to the dynamic values for flow at $\beta=0 \mathrm{deg}$, $\mathrm{Fr}=0.28$.

Longitudinal force $(X)$ for $\beta=0 \mathrm{deg}, 10 \mathrm{deg}$ and $20 \mathrm{deg}$, and side force $(Y)$ and yaw moment $(N)$ for $\beta=10 \mathrm{deg}$ and $20 \mathrm{deg}$ were measured using a load cell with data rate of $100 \mathrm{~Hz}$ for $20 \mathrm{~s}$ periods. Wave elevation $(\zeta)$ was measured using a far-field (beyond the beam) longitudinal wave-cut method with servomechanism wave probes. The data were collected at a total of 31 , 53 , and 37 cuts for $\beta=0 \mathrm{deg}, 10 \mathrm{deg}$ and $20 \mathrm{deg}$, respectively, with a fixed spacing of $\Delta y=0.01 L$ between each cut. The wave probe has a resolution of $0.1 \mathrm{~mm}\left(\Delta_{\mathrm{z}}=3.3 \times 10^{-5} L\right)$ normal to the free-surface and $1 \mathrm{~mm}\left(\Delta_{\mathrm{x}}=0.00033 L\right)$ along the streamwise direction. The $\beta=0$ deg far-field data show good agreement with Longo et al. [14], which also included near-field data (within the beam) upstream of the bow and downstream of the transom. Longo et al. [14] near field data are also used herein.

Flow-field velocities were measured using a towed underwater TPIV system at $10 y-z$ planes at $x=0.06,0.1,0.12,0.2,0.3,0.4$, $0.6,0.8,0.935$, and 1.0, as shown in Figs. $1(a)$ and $1(b)$. The TPIV resolution was $1 \mathrm{~mm}$ in all directions $\left(\Delta_{\mathrm{x}}=0.00033 L\right)$ and the data rate was $13 \mathrm{~Hz}$. The data were postprocessed to obtain averaged velocity field $(U, V, W)$, TKE and axial vorticity $\omega_{x}$. The averaged flow field data were obtained by ensemble averaging over ten repeat runs of $20 \mathrm{~s}$. For $\beta=0 \mathrm{deg}$, small static drift asymmetry $\beta_{\text {asym }}$ adjustments were made for improved flow field center plane symmetry, i.e., $\beta_{\text {asym }}=1 \mathrm{deg}$ for $x=0.06-0.2$, $\beta_{\text {asym }}=0.5 \mathrm{deg}$ for $x=0.3$ and 0.4 , and $\beta_{\text {asym }}=0 \mathrm{deg}$ for $x=0.6-1$.

\footnotetext{
${ }^{2}$ The Nomenclature provides the definitions of solution and N-version V\&V. The references provide the detailed derivations and discussions.
} 


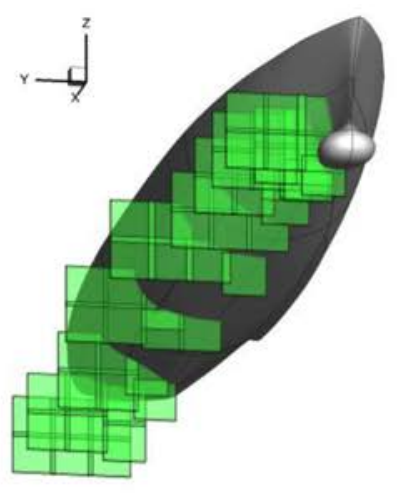

(a)

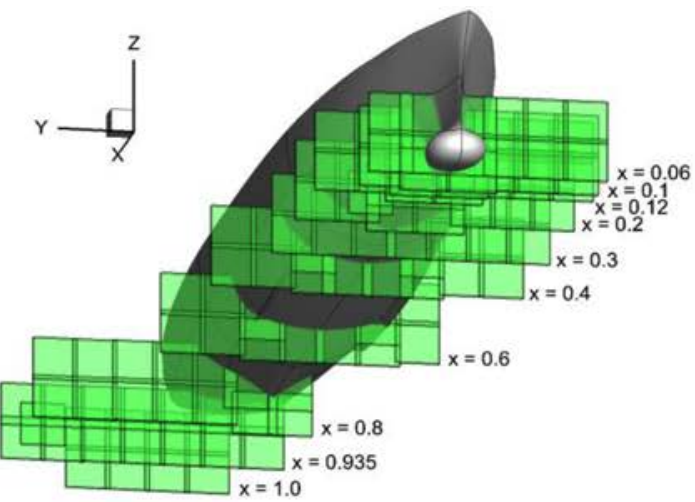

(b)

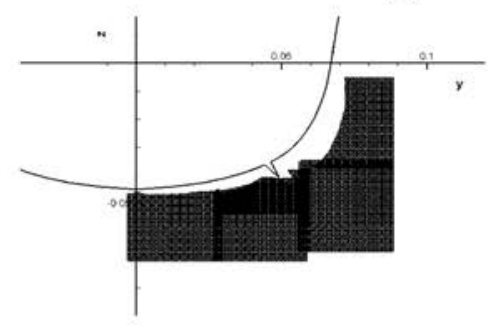

(i). EFD

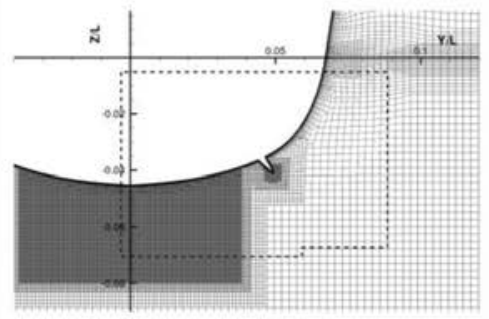

(iv). S4

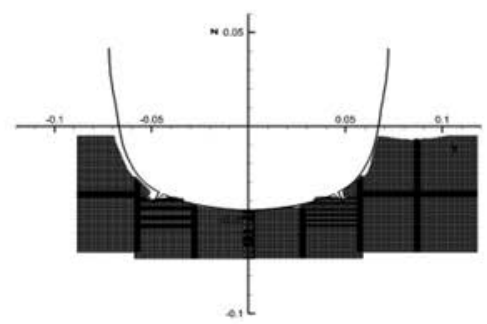

(i). EFD

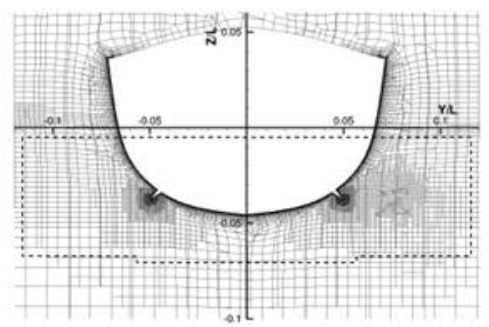

(iv). S4

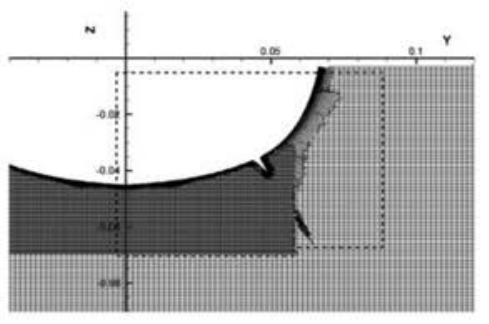

(ii). $\mathrm{S} 1 / \mathrm{S} 2$

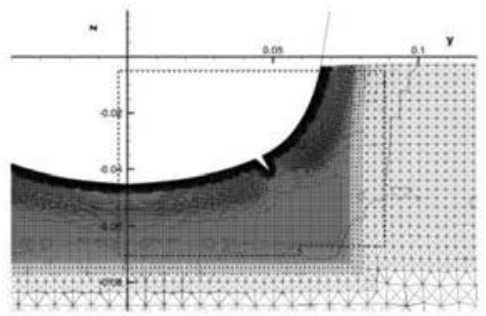

(v). S5

(c)

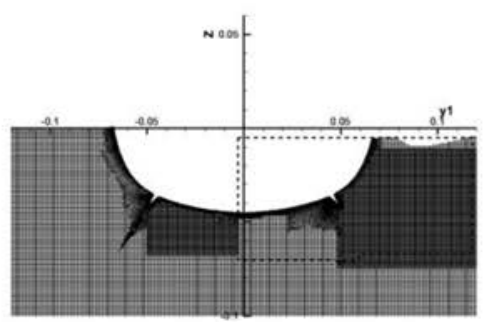

(ii). $\mathrm{S} 1 / \mathrm{S} 2$

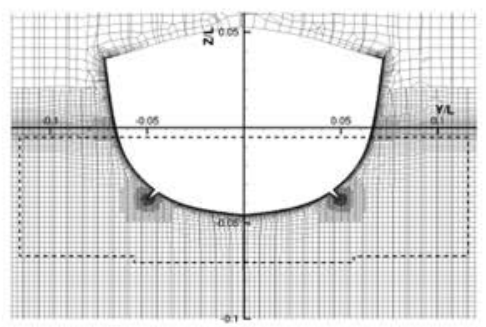

(v). S5

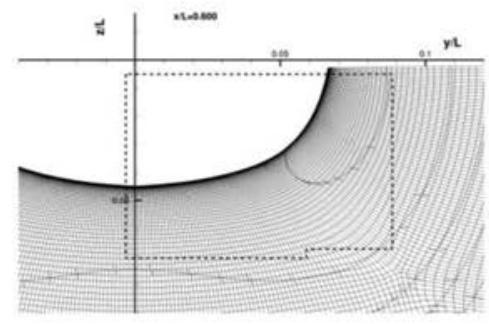

(iii). $\mathrm{S} 3$

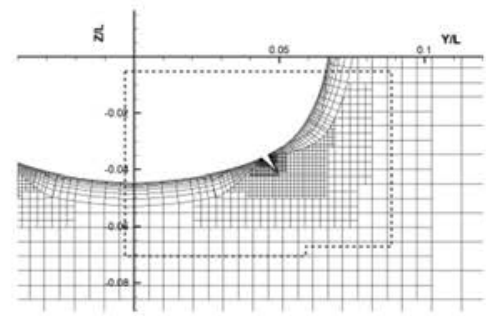

(vi). S6

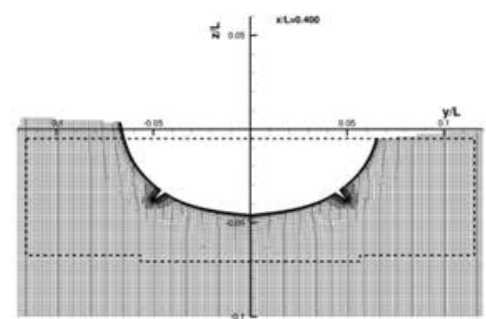

(iii). $\mathrm{S} 3$

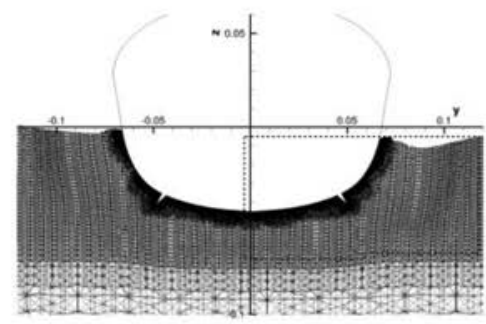

(vi). S6

Fig. 15415 geometry and TPIV measurement locations (green planes) for (a) $\beta=0 \mathrm{deg}$ and $(b) \beta=20 \mathrm{deg}$. The hull is shown at sinkage $\sigma=0$ and trim $\tau=0 \mathrm{deg}$. Grid topology used in the simulations are shown at $x=0.4$ : $(c) \beta=0$ deg and $(d) \beta=20 \mathrm{deg}$. LES zone in S2 for $(e) \beta=0 \mathrm{deg}$ and $(f) \beta=20 \mathrm{deg}$. Inset figure shows the LES zone contour at $x=0.6$. Regions flooded in red are LES zone and those in blue are URANS zone. 


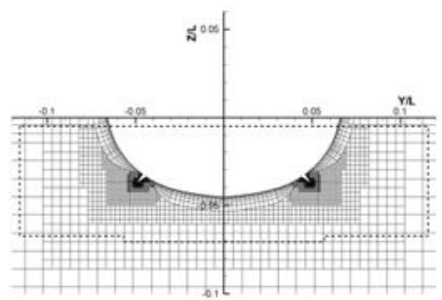

(vii). S7

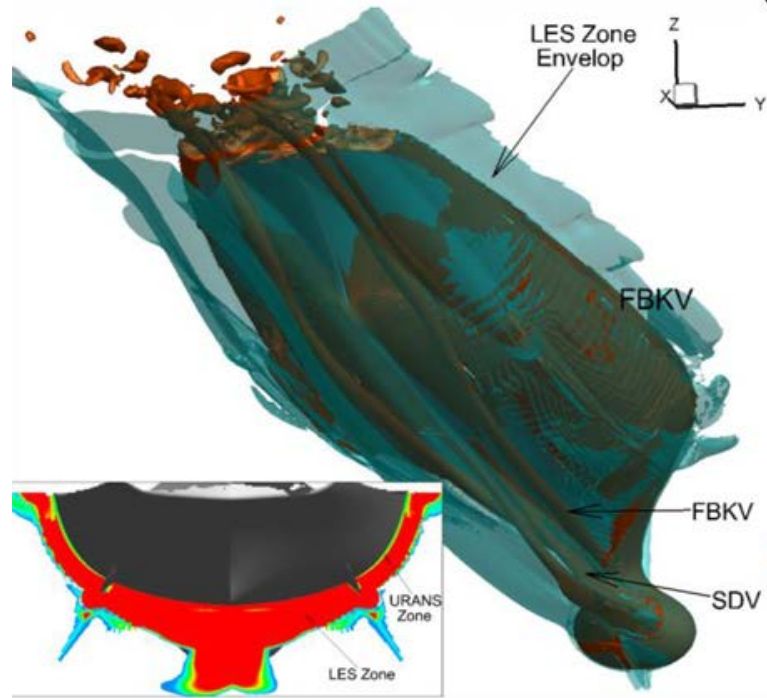

(e)

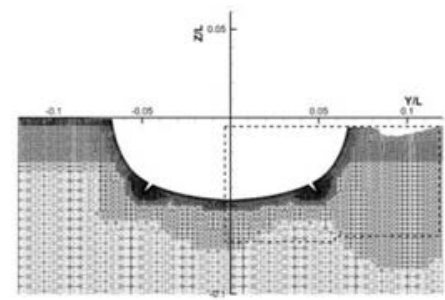

(viii). $\mathrm{S} 8$

(d)

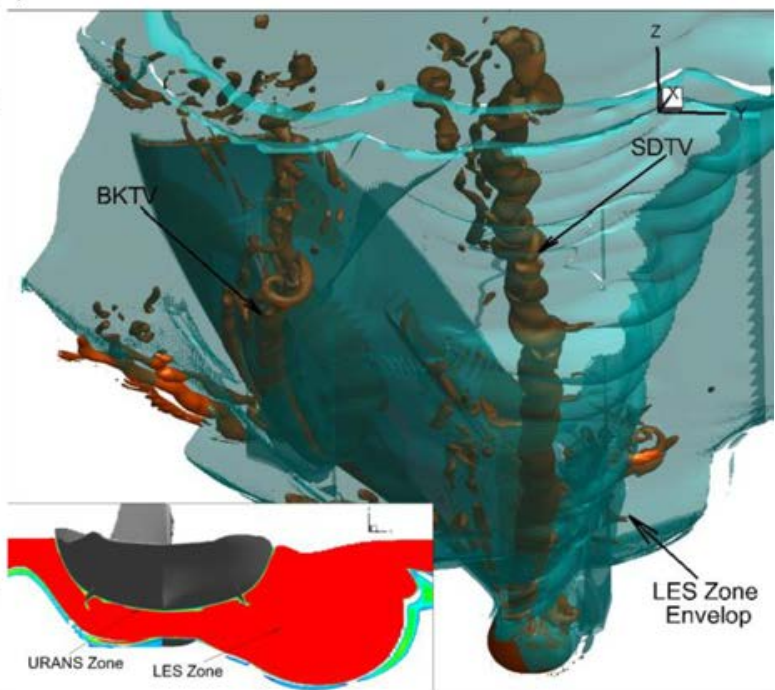

$(f)$

Fig. 1 (Continued)

Tomographic particle image velocimetry 3D volumes were analyzed along the primary vortices at all $10 y-z$ planes, namely SDV and FBKV for $\beta=0$, and sonar dome (SDTV) and bilge keel (BKTV) tip vortices for $\beta=10 \mathrm{deg}$ and $20 \mathrm{deg}$. Bhushan et al. [15] DES and URANS vortex structure predictions were used to determine the regions of interest for the TPIV measurements. The data were post processed to obtain the vorticity components $\left(\omega_{x}\right.$, $\left.\omega_{y}, \omega_{z}\right)$, second and third invariants of the deformation-rate tensor $Q$ and $\lambda_{2}$ in addition to the mean and turbulent flow variables obtained for the planar data. Unsteady analysis was performed for $\beta=20 \mathrm{deg}$ forces and moment, and velocity and $Q$ at six points along primary vortices using fast Fourier transform (FFT) of their time histories.

The experimental uncertainty $\left(U_{D}\right)$ for $X, Y$, and $N$ was $U_{D}=3.6 \%, 5.4 \%$, and $2.6 \% \mathrm{D}$, respectively. The wave-elevation and streamwise velocity $U$ measurements involved $U_{D}=5 \%$ and $2.5 \%$ for both $\beta$. The uncertainties for other velocity components were not estimated. The TKE measurements involved averaged $U_{D}=16.6 \%$ and $4.3 \%$ for $\beta=0 \mathrm{deg}$ and $20 \mathrm{deg}$, respectively. The derivative measurements (i.e., $Q$ and $\omega_{x}$ ) involved averaged $U_{D}$ $=10 \%$ and $3.2 \% D$ for $\beta=0 \mathrm{deg}$ and $20 \mathrm{deg}$, respectively. The SoA assessment tables report uncertainties for individual vortex core variables.

Herein, the data for $\beta=0 \mathrm{deg}$ and $20 \mathrm{deg}$ are used for the validation for forces and moments, wave-elevation, and axial and cross-plane vortex core predictions, and unsteady analysis for $\beta=20$ deg forces and moment and flow in the SDTV and BKTV cores. The dimensional values of forces in Newton and moment in Newton-meter are used for validation. The wave elevations, velocities, TKE, vorticity, $Q$, and instability frequencies are normalized by $L, U_{c}, U_{c}{ }^{2}, U_{c} / L, U_{c} / L$, and $L / U_{c}$, respectively, for validation.

The vortices are visualized using $Q=50$ and 100 iso-surfaces for $\beta=0 \mathrm{deg}$ and $20 \mathrm{deg}$, respectively, which was selected as the minimum value to best display the overall vortex structures. Helicity $(H)$ is used to indicate the vortex rotation direction, where $H<0$ and $>0$ correspond to clock wise and counter clockwise rotation, respectively. The peak $Q$ value is used to identify the vortex core location. $Q=10$ and 50 iso-surfaces are used to determine vortex size for $\beta=0$ and 20 , respectively. The averaged vortex radius $R_{V}$ is estimated from the on average value for vortex width along $y$ and $z$ cuts passing through the vortex core. The axial vortex core validations are performed for the prediction of core radial $(R)$ location (i.e., distance from the keel, $y=0$ and $z=0$ ), and progression of $Q, \omega_{\mathrm{x}}, U_{x}$ and TKE along the core. Cross plane validations are performed at selected locations: $x=0.2$ and 0.3 for SDV and FBKV; $x=0.2,0.4$, and 0.8 for SDTV; and $x=0.6$ for BKTV, and focused on the predictions of $R_{V}, Q, \omega_{\mathrm{x}}, U$, and TKE contours and their profiles along $y$ and $z$ cuts passing through the vortex core.

2.2 Computational Fluid Dynamics Codes, Simulation Setup, and V\&V Methods. As summarized in Table 1, the contributions numbered $1-6$ for $\beta=0 \mathrm{deg}$ and $1-8$ for $\beta=20 \mathrm{deg}$ were from six different institutions using five different codes, although results for all the validation variables were not always provided. CFDShip-Iowa from IIHR [16] and ReFRESCO from MARIN [17] are research (noncommercial) codes. ISIS-CFD from ECN/CNRS $[18,19]$ is a commercial research code distributed as FINE/Marine by NUMECA Int. STAR-CCM+ used by NTUA and FORCE are commercial codes. NavyFOAM from NSWCCD [20] is built on the open-source code OpenFOAM.

CFDShip-Iowa uses finite difference method on structured grids and level-set for free-surface modeling. The other four codes use finite volume method on unstructured grids and $\mathrm{VoF}$ for freesurface modeling. Most contributions used second-order schemes for convection, except CFDShip-Iowa DES, which used a hybrid 


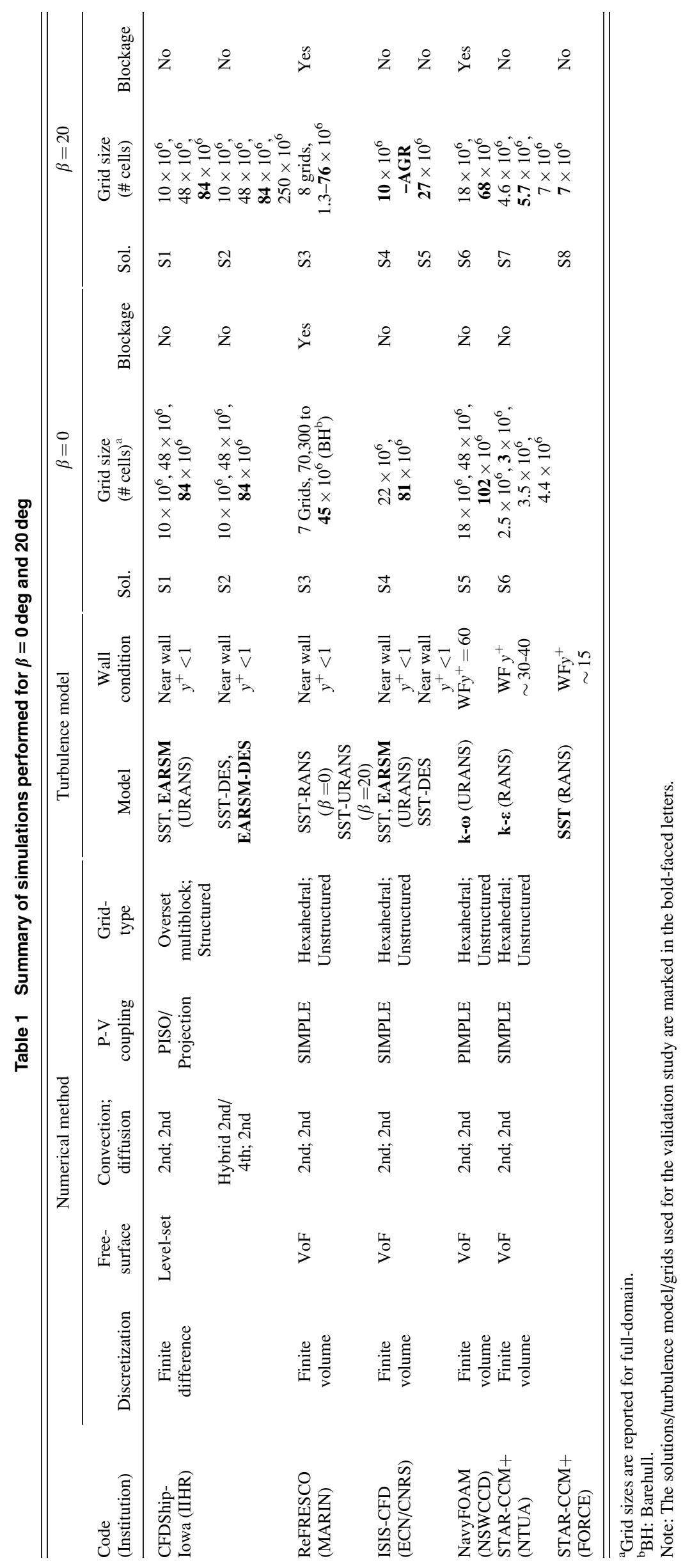


second/fourth-order scheme. All the contributions used secondorder scheme for the diffusion terms. ReFRESCO, ISIS-CFD, and STAR-CCM+ used SIMPLE scheme for pressure-velocity coupling, NavyFOAM used a coupled PISO-SIMPLE (called PIMPLE) scheme, and CFDShip-Iowa used a PISO/projection scheme.

All other contributions used hexahedral grid elements and refined the grid along the vortex cores. ISIS-CFD used automatic grid refinement (AGR) using local Hessian of pressure. Others used manual grid refinement based on coarse grid solutions using both overset block grids and local grid refinement. Figures 1(c) and $1(d)$ show cross sections of the measurement and simulation grids for $\beta=0 \mathrm{deg}$ and $20 \mathrm{deg}$, respectively. All the contribution solved for water phase along with the free-surface, except ReFRESCO for $\beta=0 \mathrm{deg}$. The latter prescribed the top boundary as deformed water surface obtained from a separate computation with $\mathrm{VoF}$ free-surface model.

Most contributions performed simulations on multiple grids to assess the grid sensitivity of results, but only CFDShip-Iowa and ReFRESCO reported quantitative grid uncertainty. For $\beta=0 \mathrm{deg}$, all except one used at least one grid finer than $45 \mathrm{M}$ cells, and were significantly finer than those in G2010. For $\beta=20 \mathrm{deg}$, half of the contributions used grids with about $10 \mathrm{M}$ cells and rest used $>70 \mathrm{M}$ cells. The finest grids for both $\beta$ used cubic cells in the vortex core with grid resolutions $\Delta_{\mathrm{x}}=0.00122 \mathrm{~L}$. The grids around the free-surface used anisotropic cells with 1:6 aspect ratio, where grids are finest along the free-surface normal direction with cells was $\Delta_{\mathrm{z}}=2.5 \times 10^{-4} L$. The grid resolution are about four times $(\times)$ coarser than the experimental resolution in the vortex core. For the free-surface, the streamwise grid resolution is comparable to the experiment, but the resolution normal to the free-surface is and $10 \times$ coarser. CFDShip-Iowa and ReFRESCO simulations used time-step size $\Delta \mathrm{t}=2.5 \times 10^{-4} L / U_{0}$ and $1.5 \times 10^{-2} L / U_{0}$, respectively, where $U_{0}$ is the inflow free-stream water velocity used in the simulation and is same as $U_{c}$. The smallest frequency resolved in CFDShip-Iowa and ReFRESCO simulations were $\sim 8000 \mathrm{~Hz}$ and $\sim 166 \mathrm{~Hz}$, respectively. Others did not report their time-step size. But considering that the unsteady simulations usually require Courant-Friedrichs- Lewy condition $<1$, all the simulations resolved smallest frequencies $>100 \mathrm{~Hz}$ resolved in the experiments. For DES, Courant-Friedrichs-Lewy $\sim 0.2$; thus, time-step size was small enough to resolve the coherent turbulent structures larger than the grid size.

CFDShip-Iowa and ISIS-CFD contributions investigated the effects of isotropic and anisotropic URANS and DES turbulence models, and ReFRESCO contribution investigated different anisotropic RANS/URANS turbulence models. CFDShip-Iowa and ISIS-CFD used isotropic $k-\omega$ shear stress transport (SST) and anisotropic explicit algebraic (EARSM) URANS [21] and DES [22] models with near-wall resolution $\left(y^{+}<1\right)$. ReFRESCO used different variants of $k-\omega$ RANS for $\beta=0$ deg and $k-\omega$ URANS for $\beta=20$ deg models with near-wall resolution $[21,23]$. NavyFOAM used $k-\omega$ URANS model with wall functions (WF) for wall resolution $y^{+} \sim 60$. STAR-CCM+ used $k-\varepsilon$ or $k-\omega$ SST RANS turbulence model with WF for wall resolution $y^{+} \sim 15-40$. ReFRESCO simulations included tow-tank sidewalls (blockage) for both $\beta$. NavyFOAM $\beta=20 \mathrm{deg}$ submissions included simulations using both with and without sidewalls.

The $\beta=0 \mathrm{deg}$ discussions are based on predictions from: CFDShip-Iowa using EARSM (S1) and DES (S2) on 84 M grid; ReFRESCO using SST-RANS on $45 \mathrm{M}$ grid (S3) for the bare hull; ISIS-CFD using EARSM on $10 \mathrm{M}-\mathrm{AGR}$ grid (S4); NavyFOAM using $k-\omega$ on $102 \mathrm{M}$ grid (S5); and STAR-CCM+ (NTUA) using $k-\varepsilon$ on $5.7 \mathrm{M}$ grid (S6). The $\beta=20 \mathrm{deg}$ discussions are based on predictions from: CFDShip-Iowa using EARSM (S1) and DES (S2) on $84 \mathrm{M}$ grid; ReFRESCO using SST-URANS on $76 \mathrm{M}$ grid (S3); ISIS-CFD using EARSM on $81 \mathrm{M}$ grid (S4) and SST-DES on $27 \mathrm{M}$ grid (S5); NavyFOAM using $k-\omega$ on $68 \mathrm{M}$ grid (S6); STAR-CCM+ (NTUA) using $k-\varepsilon$ on $3 \mathrm{M}$ grid (S7); and STAR$\mathrm{CCM}+($ FORCE) using $k-\omega$ on $7 \mathrm{M}$ grid (S8).
For $\beta=0 \mathrm{deg}$, all the solutions were steady, including S2 DES, which is unexpected, but similar behavior was reported in G2010. Figure 1(e) shows that for S2 DES, even though LES is activated in the vortex onset and progression regions, the resolved turbulence is $<3 \%$ of the total TKE. Thus, the flow exhibits laminarlike behavior. The low resolved TKE level is probably due to modeled stress depletion issue in DES. The absence of resolved turbulence causes the flow to be steady, and the vortices are intensified. However, the resistance and wave pattern predictions are accurate, as URANS in activated in the boundary layer and the free-surface deformation is primarily an inviscid phenomenon. In addition, it provides a plausible description of the overall vortex structures. Thus, although the turbulence is under resolved the solution is still valuable.

For $\beta=20 \mathrm{deg}$, URANS solution S1 and DES solutions S2 and S5 were unsteady, whereas the others were steady. S1 mostly predicted large-scale oscillations, but S2 and S5 predicted smallscale vortical structures expected to be resolved turbulence. In S2, LES was active in the separated flow and vortex progression regions, and URANS in the boundary layer and outside the separated flow region (Fig. 2(f)). The peak-resolved TKE was observed in the vortex core region, and increased with grid refinement and is estimated to be $60 \%, 80 \%$, and $95 \%$ of total TKE on $10 \mathrm{M}, 48 \mathrm{M}$, and $84 \mathrm{M}$ cell grids, respectively. S5 reported dominant modeled turbulence up to $x=0.4$, and dominant resolved turbulence for $x>0.6$.

Solution verification focuses on $U_{S N i}$ and its components. Solution validation focuses on $U_{V i}$ and its components, $E_{i}$ and its sign, and $\left|E_{i}\right|$, and whether errors are $<$ or $>U_{V i}$. N-version verification focuses on the code/solution scatter $\sigma_{S}$. N-version validation focuses on $U_{S O A i}$ and $U_{S O A}$ and their components, and $\left|E_{i}\right|$ and $\overline{|E|}$ and whether errors are $<$ or $>U_{S O A i}$ and $U_{S O A}$, respectively. The relative contributions of the uncertainty components are useful for identification of resource allocation for reduction of the solution and $\mathrm{N}$-version validation intervals.

2.3 Solution Verification. CFDShip-Iowa estimated numerical uncertainties $U_{S N_{i}}$ for forces and moment, wave-elevation and vortex core predictions using the factor of safety method for grid triplets with refinement ratio $r_{G}=\sqrt{2}$. ReFRESCO estimated $U_{S N_{i}}$ for forces and moment using least square method. Table 2 shows the average $U_{\overline{S N}}$ values for forces and moment used for the validation of all codes/simulations. CFDShip-Iowa $U_{S N_{i}}$ values for freesurface and vortex core prediction are shown in Tables 2 and 4 , respectively, and are used for validation of all codes/simulations.

For forces and moment, both studies reported negligible iterative errors $\left(U_{I}=0.01 \% S_{i}\right)$ compared to grid uncertainty $U_{G i}$. $X$ solutions showed monotonic convergence, whereas $Y$ and $N$ solutions showed mixed monotonic and oscillatory convergence. For both $\beta$, URANS estimated order of accuracy ratio $P \sim 1$ suggesting that the grids are close to the asymptotic range. Whereas DES estimated large $P \geq 2$ suggesting that even finer grids are required to achieve the asymptotic range. For $\beta=0 \mathrm{deg}$, averaged $U_{S N_{i}}=2 \% S_{i}$ for URANS and $\sim 1 \% S_{i}$ for DES, which are lower than $U_{S N_{i}}=3 \% S_{i}$ reported in G2010 (on 2-10 M cells) because of the use of finer grids. For $\beta=20 \mathrm{deg}$, both URANS and DES show similar uncertainties, and $U_{\overline{S N}}=1.4 \% \bar{S}$ comparable to $\beta=0 \mathrm{deg}$.

For wave elevation, $U_{I}=0.03 \% S_{i}$ is somewhat larger than that for integral variables, but significantly small compared to $U_{G i}$. Both URANS and DES, and both $\beta$ showed monotonic convergence with averaged $P \sim 0.65$. This suggests that finer grids are required to achieve the asymptotic range, $P=1$. URANS and DES showed similar uncertainties, and $U_{\overline{S N}}=3.5 \%$ and $5.8 \% \bar{S}$ for $\beta=0 \mathrm{deg}$ and $20 \mathrm{deg}$, respectively.

For the vortex core variables, $U_{I}=0.2 \% S_{i}$ is an order of magnitude larger than that for integral variables, but small compared to $U_{G i}$. URANS predicted mostly monotonic convergence with averaged $P \sim 0.3$, which suggests that grids are far from the asymptotic range. DES predicted mixed oscillatory and monotonic 


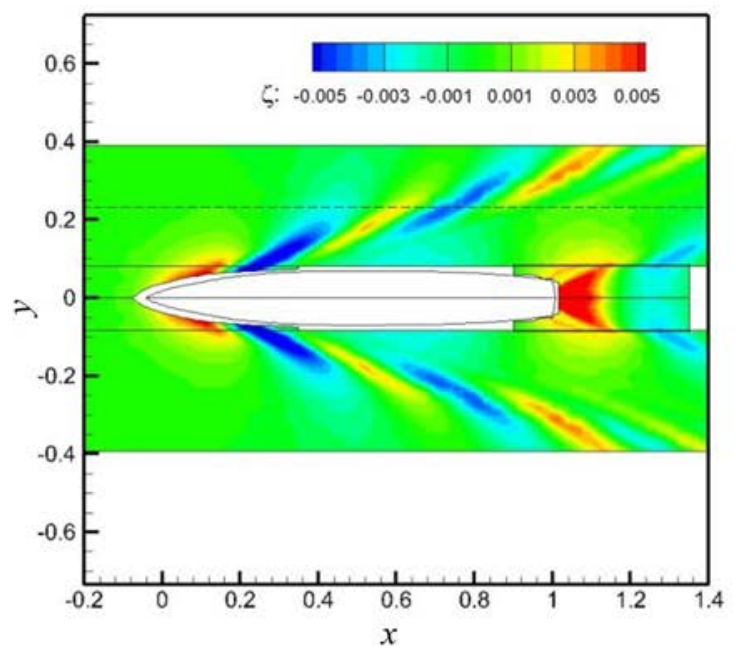

(a)

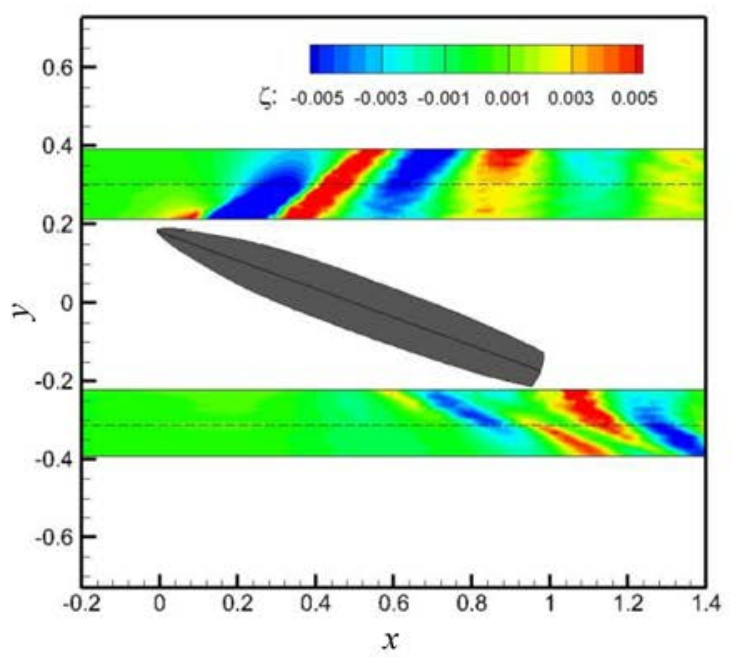

(c)

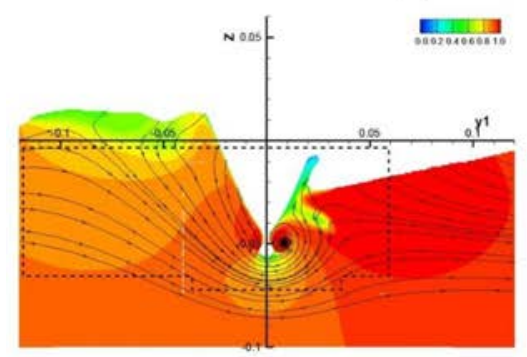

(i) Solution \#2

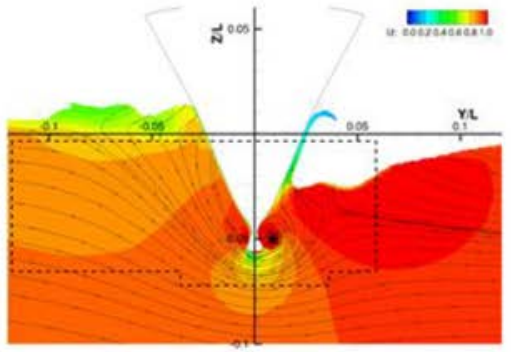

(ii). Solution \#4

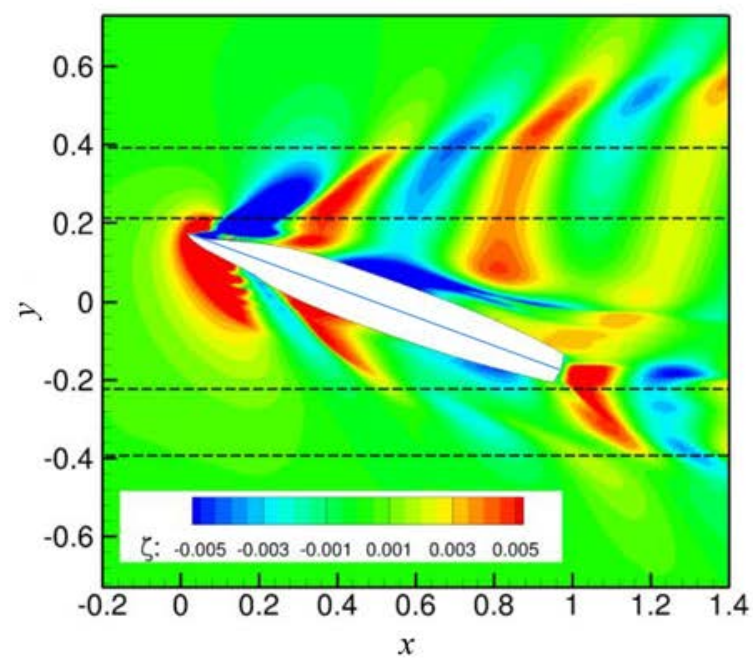

(d)

(e)

Fig. 2 Wave-elevation contours obtained using: (a) experiment and (b) S3 for $\beta=0$ deg; (c) experiment and (d) S4 for $\beta=20$ deg. (e) Wave elevation pattern predicted by solution \#2 (using level set) and S4 and S6 (both using VoF) at $x=0.1$ shows the differences in wave breaking pattern on the leeward sonar dome.

convergence, and about $5 \%$ higher $U_{G i}$ than URANS. The averaged $U_{\overline{S N}}$ varied from $10.5 \% \bar{S}$ for SDV to $20 \% \bar{S}$ for SDTV.

\section{Forces and Moment, and Wave Elevation}

3.1 Straight-Ahead $\boldsymbol{\beta}=\mathbf{0}$ deg. Table 2 summarizes the SoA assessment results for resistance and wave elevation for $\beta=0 \mathrm{deg}$.
For $X$, four out of six submissions overpredict and two underpredict. At the solution level, $U_{V i}=4 \% D$ with $U_{D}$ more than twice of $U_{\overline{S N}}$. Thus, reduction in $U_{D}$ is most important. The mean code $\bar{E}=1 \% D$, i.e., dominated by the two under predictions, whereas $\overline{|E|}=4 \% D$. The latter is a better indication of the average code/ simulation capability. The four solutions that overpredict are validated, whereas two that under predict are not. At the code level, the scatter $\sigma_{S}=4 \% \bar{S}$ is similar to $U_{V i}$. $U_{S o A i}=6 \% D$ and largest 
Table 2 CFD SoA assessment of forces and moment and wave-elevation for $\beta=0$ deg and 20 deg

\begin{tabular}{|c|c|c|c|c|c|c|c|c|c|}
\hline \multicolumn{2}{|l|}{ Case } & \multicolumn{3}{|c|}{$\beta=0 \mathrm{deg}$} & \multicolumn{5}{|c|}{$\beta=20 \mathrm{deg}$} \\
\hline \multicolumn{2}{|l|}{ Evaluations $\Rightarrow$} & \multirow[b]{2}{*}{ Sol. } & \multirow[b]{2}{*}{$\begin{array}{c}X \\
E_{i} \% D\end{array}$} & \multirow[b]{2}{*}{$\begin{array}{c}\text { Wave-elevation } \\
\left\langle E_{i}\right\rangle \% D\end{array}$} & \multirow[b]{2}{*}{ Sol. } & \multicolumn{3}{|c|}{ Forces and moment } & \multirow[b]{2}{*}{$\begin{array}{c}\text { Wave-elevation } \\
\left\langle E_{i}\right\rangle \% D\end{array}$} \\
\hline Solution $\Downarrow$ & & & & & & $\begin{array}{c}X \\
\left(E_{i} \% D\right)\end{array}$ & $\begin{array}{c}Y \\
\left(E_{i} \% D\right)\end{array}$ & $\begin{array}{c}N \\
\left(E_{i} \% D\right)\end{array}$ & \\
\hline \multirow[t]{2}{*}{ Experiment } & $D(N$ or $N-m)\left(\times 10^{-3}\right)$ & & -16.63 & Fig. 1(a) & & -38.57 & 153.57 & 59.86 & Fig. 1(c) \\
\hline & $U_{D} \% D$ & & 3.6 & 5.0 & & 3.6 & 5.4 & 2.6 & 5.0 \\
\hline \multirow[t]{2}{*}{ Solution V\&V } & $U_{\overline{S N}} \% \bar{S}$ & & 1.72 & 3.5 & & 1.5 & 1.6 & 1.3 & 5.8 \\
\hline & $U_{V_{i}}^{S N} \% D$ & & 4.0 & 6.1 & & 3.9 & 5.6 & 2.9 & 7.7 \\
\hline \multirow[t]{8}{*}{ Solutions } & & S1 & -1.36 & 4.28 & S1 & -5.1 & -3.8 & -0.6 & 6.91 \\
\hline & & $\mathrm{S} 2$ & -1.74 & 4.32 & $\mathrm{~S} 2$ & -4.5 & -3.1 & -0.25 & 6.85 \\
\hline & & S3 & $-0.06^{\mathrm{a}}$ & 2.1 & $\mathrm{~S} 3$ & -5.7 & 2.0 & 0.4 & 11.8 \\
\hline & & S4 & 6.2 & 3.2 & S4 & 0.35 & -4.2 & -0.18 & 6.5 \\
\hline & & S5 & 5.7 & 6.0 & S5 & 1.78 & 0.22 & -1.70 & 6.5 \\
\hline & & S6 & -1.0 & - & S6 & 4.5 & -6.5 & 7.0 & 12.0 \\
\hline & & & & & S7 & 1.4 & -0.3 & 4.0 & \\
\hline & & & & & S8 & -13.1 & -4.6 & -5.4 & \\
\hline \multirow[t]{2}{*}{$\mathrm{N}-$ version verification } & \#Submission & & 6 & 5 & & 8 & 8 & 8 & 6 \\
\hline & $\sigma_{S}=\sigma_{E} \% \bar{S}$ & & 3.96 & 1.5 & & 5.65 & 2.87 & 3.7 & 2.6 \\
\hline \multirow[t]{6}{*}{$\mathrm{N}$-version validation } & $\bar{E} \% D$ & & 1.29 & - & & -2.55 & -2.53 & 0.41 & - \\
\hline & $\overline{|E|} \% D$ & & 3.67 & 3.82 & & 4.55 & 3.09 & 2.44 & 9.3 \\
\hline & $P_{\left|E_{i}\right|}$ & & 4.6 & 3.0 & & 7.42 & 4.02 & 5.0 & 5.2 \\
\hline & $P_{\overline{|F|}}^{||_{i} \mid}$ & & 1.9 & 1.3 & & 2.6 & 1.4 & 1.8 & 2.1 \\
\hline & $U_{S o A} \% D$ & & 6.2 & 6.8 & & 8.6 & 5.7 & 6.6 & 9.3 \\
\hline & $U_{S o A} \% D$ & & 4.4 & 6.3 & & 5.0 & 4.5 & 4.6 & 8.1 \\
\hline \#validated & & & 4 & 5 & & 3 & 7 & 5 & 4 \\
\hline \#N-version validated & & & 6 & 5 & & 7 & 7 & 7 & 4 \\
\hline Mean code $\mathrm{N}$-version $\mathrm{v}$ & idated & & Yes & Yes & & Yes & Yes & Yes & No \\
\hline
\end{tabular}

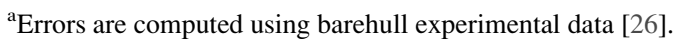

contributions are from $P_{\left|E_{i}\right|}$ and $U_{D}$. $U_{S o A}=4 \% D$ and largest contribution is from $U_{D}$. Thus, all codes/simulations and mean code are $\mathrm{N}$-version validated.

There are five contributions for wave elevation. Figures 2(a) and $2(b)$ show the wave patterns obtained in the experiment and from S3, respectively. S3 is used as a representative CFD plot as it shows smallest average error All codes/solutions qualitatively predicted the wave pattern, with symmetric diverging waves emerging from the bow with a wave envelop angle $\alpha=16.5 \mathrm{deg}$. The errors were smallest for the solution with the finest far-field grid resolution. Simulations did not display wave breaking and induced vortices. At the solution level, $U_{V i}=6 \% \mathrm{D}$ with $U_{D} \sim 1.4 \times U_{\overline{S N}}$ and $\overline{|E|}=4 \% D$. All of the solutions are validated. At the code level, the scatter $\sigma_{S}=1.5 \% \bar{S}$, which is one-fourth of $U_{V i} . U_{S o A}=7 \% D$ and largest contribution is from $U_{D}$. $U_{S o A}=6 \% D$ and largest contribution is from $U_{D}$. All codes/ simulations and mean code are $\mathrm{N}$-version validated.

Static Drift $\beta=20 \mathrm{deg}$. Table 2 includes SoA assessment for forces and moment and wave elevation for the $\beta=20 \mathrm{deg}$. There are eight contributions for $X, Y$, and $N$, and six contributions for wave elevation. $X$ and $Y / N$ versus $\beta$ increase following quadratic and cubic polynomials, as expected.

For $X$ at the solution level, $U_{V i}=4 \% D$ with $U_{D}$ more than twice of $U_{\overline{S N}}$. Half of the solutions overpredict and half underpredict. The mean code $\bar{E}=-2.6 \% D$ is dominated by the four over predictions, whereas $\overline{|E|}=4.6 \% \mathrm{D}$. Only three of the solutions are validated. At the code level, the scatter $\sigma_{S}=5.7 \% \bar{S}$ is larger than $U_{V i} . U_{S o A}=8.6 \% D$ with $P_{\left|E_{i}\right|}>U_{V i} . U_{S o A}=5 \% D$ with similar contribution from $U_{V i}$ and $P \frac{\mid E_{|E|}}{|c|}$. All but one code/simulation and mean code are $\mathrm{N}$-version validated. For $Y$ at the solution level, $U_{V i}=6 \% D$ with $U_{D}$ more than three times of $U_{\overline{S N}}$. Six solutions overpredict and two underpredict. The mean code $\bar{E}=-2.5 \% D$ is dominated by the six over predictions, whereas $\overline{|E|}=3 \% D$. All but one solution is validated. At the code level, the scatter $\sigma_{S}=3 \% \bar{S}$ is half of $U_{V i} . U_{S o A i}=6 \% D$ with similar contribution from $U_{D}$ and $P_{\left|E_{i}\right|} . U_{S o A}=5 \% D$ and largest contribution is from $U_{D}$. All but one code/simulation and mean code are $\mathrm{N}$-version validated.

For $N$ at the solution level, $U_{V i}=3 \% D$ with $U_{D}$ twice of $U_{\overline{S N}}$. Five solutions overpredict and three underpredict. The mean code $\bar{E}=0.41 \% D$, whereas $\overline{|E|}=2.4 \% D$. Five solutions are validated and three are not. At the code level, the scatter $\sigma_{S}=4 \% \bar{S}$, which is somewhat larger than $U_{V i} . U_{S o A i}=7 \% D$ with $P_{\left|E_{i}\right|}>U_{V i}$. $U_{S o A}=5 \% D$ with similar contribution from $U_{D}$ and $P_{\overline{|E|}}$. All but one code/simulation and mean code are $\mathrm{N}$-version validated.

Figures 2(c) and 2(d) show the wave patterns obtained in the experiment and from $\mathrm{S} 4$, which shows the smallest average error, respectively. The qualitative agreement is good. The wave pattern is asymmetric with $\alpha=3 \mathrm{deg}$ and $32.5 \mathrm{deg}$ with respect to the hull axis on the windward and leeward sides, respectively. The total wave envelop angle of $35.5 \mathrm{deg}$ is close to $37 \mathrm{deg}$ observed for $\beta=0$ deg. Longo and Stern [24] observed similar asymmetric wave patterns for the Series 60 at $\beta=5 \mathrm{deg}$ and $10 \mathrm{deg}$ with total wave envelope angle of $38 \mathrm{deg}$ close to the $\beta=0 \mathrm{deg}$ value. The simulations show wave breaking on the windward bow (Fig. 2(e)), SDTV induced breaking wave scar on the leeward side (Fig. $2(d)$ ), and in the transom region due to transom vortices. Leeward bow and shoulder wave also show secondary breakings. VoF predictions, S4, S5, and S6, show very deep trough at leeward bow compared to level-set predictions, S1 and S2. The deep trough was region of bubbly mixture formed by wave breaking. In addition, VoF predicts thin water sheet attached to the hull, but level-set does not predict such features. Thin water sheets have been observed for the series 60 at $\beta=0$ deg that extend above the bow wave [25]. They were analyzed using detailed point gauge measurements, and attributed to surface tension effects. At the solution level, $U_{V i}=8 \% D, U_{\overline{S N}}$ is larger than $U_{D}$, and $\overline{|E|}=9 \% D$. Four out of six codes/solutions are validated. At the code level, the scatter $\sigma_{S}=3 \% \bar{S}$, which is one-third of $U_{V_{i}} . U_{S o A}=9 \% D$ with similar contributions from $U_{\overline{S N}}, U_{D}$ and $P_{\left|E_{i}\right|} \cdot U_{S o A}=8 \% D$ with 
Table 3 CFD SoA assessment of axial vortex core for $\beta=0 \mathrm{deg}$ and $20 \mathrm{deg}$

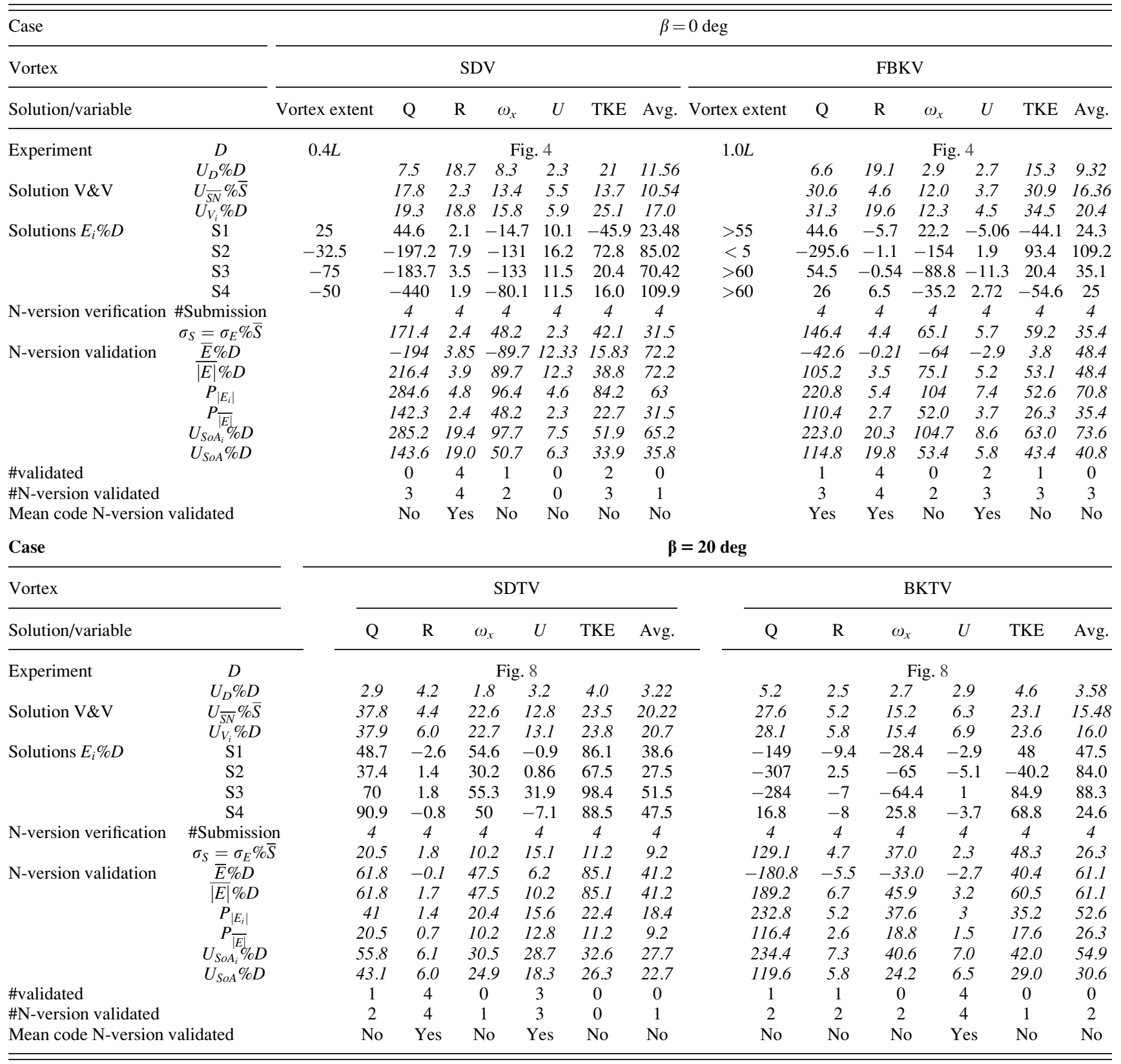

Table 4 Verification and validation of cross-plane vortex core predictions for $\beta=0$ deg and $20 \mathrm{deg}$

\begin{tabular}{|c|c|c|c|c|c|c|c|c|c|c|c|c|c|}
\hline \multirow{3}{*}{$\begin{array}{l}\text { Case } \\
\text { Vortex } \\
\text { Solution/variable }\end{array}$} & & \multicolumn{12}{|c|}{$\beta=0 \mathrm{deg}$} \\
\hline & & \multicolumn{6}{|c|}{ SDV } & \multicolumn{6}{|c|}{ FBKV } \\
\hline & & Size & Q & $\omega_{x}$ & $U$ & TKE & Avg. & Size & Q & $\omega_{x}$ & $U$ & TKE & Avg. \\
\hline $\begin{array}{l}\text { Solution V\&V } \\
\text { Solutions }\left\langle E_{i}\right\rangle \% D\end{array}$ & $\begin{array}{c}U_{V_{i}} \% D \\
\text { S1 } \\
\text { S2 }\end{array}$ & $\begin{array}{c}19.3 \\
26 \\
12.9\end{array}$ & $\begin{array}{l}19.3 \\
44.4 \\
29.7\end{array}$ & $\begin{array}{l}15.8 \\
38.3 \\
82.4\end{array}$ & $\begin{array}{l}5.9 \\
3.4 \\
7.5\end{array}$ & $\begin{array}{l}25.1 \\
54.8 \\
76.1\end{array}$ & $\begin{array}{l}17.0 \\
33.4 \\
41.7\end{array}$ & $\begin{array}{c}31.3 \\
12.7 \\
14\end{array}$ & $\begin{array}{l}31.3 \\
69.7 \\
75.7\end{array}$ & $\begin{array}{c}12.3 \\
38 \\
55.8\end{array}$ & $\begin{array}{r}4.5 \\
10 \\
11\end{array}$ & $\begin{array}{l}34.5 \\
52.8 \\
63.6\end{array}$ & $\begin{array}{c}20.4 \\
36.7 \\
44\end{array}$ \\
\hline Case & & \multicolumn{12}{|c|}{$\beta=20 \mathrm{deg}$} \\
\hline Vortex & & \multicolumn{6}{|c|}{ SDTV } & \multicolumn{6}{|c|}{ BKTV } \\
\hline Solution/va & & Size & Q & $\omega_{x}$ & $U$ & TKE & Avg. & Size & $\mathrm{Q}$ & $\omega_{x}$ & $U$ & TKE & Avg. \\
\hline $\begin{array}{l}\text { Solution V\&V } \\
\text { Solutions }\left\langle E_{i}\right\rangle \% D\end{array}$ & $\begin{array}{c}U_{V_{i}} \% D \\
\mathrm{~S} 1 \\
\mathrm{~S} 2\end{array}$ & $\begin{array}{c}37.9 \\
121.3 \\
56.1\end{array}$ & $\begin{array}{l}37.9 \\
95.9 \\
63.7\end{array}$ & $\begin{array}{l}22.7 \\
58.2 \\
19.2\end{array}$ & $\begin{array}{l}13.1 \\
19.9 \\
17.3\end{array}$ & $\begin{array}{c}23.8 \\
51 \\
31.6\end{array}$ & $\begin{array}{l}20.7 \\
69.3 \\
37.6\end{array}$ & $\begin{array}{l}28.1 \\
14.6 \\
21.4\end{array}$ & $\begin{array}{l}28.1 \\
62.2 \\
46.9\end{array}$ & $\begin{array}{l}15.4 \\
60.5 \\
12.7\end{array}$ & $\begin{array}{c}6.9 \\
4.1 \\
15.6\end{array}$ & $\begin{array}{l}23.6 \\
93.2 \\
68.7\end{array}$ & $\begin{array}{c}16.0 \\
46.9 \\
33\end{array}$ \\
\hline
\end{tabular}


$U_{V i}>P_{\overline{|E|}}$. Four codes/simulations are N-version validated, but the mean code is not.

\section{Overall Vortex Structures, Onset, and Progression}

4.1 Straight-Ahead $\boldsymbol{\beta}=\mathbf{0}$ deg. Figure 3 shows the vortical structures observed in the experiment and CFD results using isosurfaces of $Q$ colored by $H$. Zoomed in view shows more detail of the vortex onset and progression. The flow is symmetric with respect to the center plane such that the port (PS) and starboard (SS) side vortex pairs have antisymmetric rotation. The downward/inward flow on the fore body generates the primary vortices due to the sonar dome and secondary vortices due to the bilge keels with clockwise and counter clockwise rotation on the PS and SS, respectively.

As shown in Fig. 3(a), experiment data clearly display SDV, FBKV, and bilge keel vortex (BKV). The first instance of SDV observation is at $x=0.1$, originating parallel to the upper surface of the sonar dome and aligned at an angle of about $20 \mathrm{deg}$ with respect to the center plane. At $x=0.12$ (trailing edge of sonar dome), SDV progresses downward (away from hull), and FBKV is first observed originating parallel to the hull bottom and above SDV. Zoomed in views indicate onset of sonar dome trailing edge vortex (SDTEV). SDV and FBKV progress at $x=0.2,0.3,0.4$ and 0.6 with SDV in the outer and FBKV in the inner boundary layer. SDV strength reduces rapidly such that $Q<25$ by $x=0.6$, whereas FBKV retains large $Q>50$ values up to $x=1$. BKV is first observed at $x=0.6$. The weak SDV and BKV and strong FBKV progress and interact at $x=0.8,0.935$ and 1 , resulting in thinning/thickening of the boundary layer near the center plane/ free-surface causing mid-girth bulge similar to the interaction of common down vortex pair with boundary layer. The experiments confirm Olivieri et al. [26] five-hole pitot measurements that showed presence of presence of two corotating vortices at $x=0.6$, i.e., SDV and FBKV, including their orientation/location. However, SDV in Olivieri et al. [26] measurements was more prevalent than the present measurements, which may be due to scale effects and/or $\beta_{\text {asym }}$. The present measurements also show good agreement with previous two-dimensional (2D) and stereo PIV measurements at nominal wake plane [14]. However, the data are too sparse to clarify the physics of vortex progression and interaction on the after-body.

As shown in Figs. 3(b) and 3(c), S2 clearly displays the overall vortex structures, but the results should be taken into consideration with caution as turbulence is under resolved. S2 also shows midhull vortex (MHV), after-body keel vortex (ABKV), and transom vortices. BKV indicates vortex pairs with inboard/outboard clockwise/counterclockwise rotation on the SS. The trends are qualitatively similar to the experiments; however, the $Q /$ vortex strength and TKE progression are overpredicted and underpredicted, respectively. S1 shows better prediction of the vortex progression than $\mathrm{S} 2$; however, with higher decay such that $Q<30$ for SDV beyond $x=0.4$ and for FBKV beyond $x=0.6$. S3, S4, and S5 predict vortices not only similar to S1 and S2 but also with higher decay similar to S1. In addition, they show SDV is stronger and persists longer than FBKV contrary to the experiments and S1 and S2. Interestingly, S4 on 22M point grid (results not shown) does indicate correct trend that FBKV is stronger and persists longer than SDV. S4 and S5 also show wave-induced vortices near the free-surface.

The onset of the vortex separations was identified by tracking the $Q=10$ iso-surfaces up to the hull surface. All solutions showed agreement for both the onset location and surface streamline pattern predictions. SDV, SDTEV, FBKV, and MHV onset occurred at $x=0.065,0.109,0.107$, and 0.5 , respectively. The surface streamlines showed converging streamlines close to the vortex onset; thus, it is crossflow/open type separation [27]. Simpson et al. [28] reported that open-type separation occurs as the crossflow pressure gradient induces streamline convergence, which along with adverse axial pressure gradient induces high normal velocity lifting the vortex off the hull surface. They estimated that the normal velocity satisfied the relation

$$
V_{n}=-\frac{1}{2 \mu}\left(\nabla \cdot \tau_{w}\right) y^{2}+\frac{1}{6 \mu}\left(\nabla^{2} P\right) y^{3}
$$

where $y$ is the distance from the wall and $\tau_{w}$ is the wall shear stress. The above relation suggests that the second term is much smaller than the first as $y \rightarrow 0$; thus, vortex separation location is expected to occur near the peak $\nabla \cdot \tau_{w}$. The results in Fig. 4 for SDV show separation pattern similar to the above mechanism. BKV also showed converging streamlines at the bilge keel tip. The vortex also shows open-type separation wherein vortex sheet emerges from the separation line at the sharp edge of BKV.

Figure 5 shows the streamwise variation of the SDV and FBKV core variables. Plots show experimental data, S1-S4, $\bar{S}, \sigma_{S}$ and $D$, and $R, \omega_{\mathrm{x}}$, and $U$ from experiment [26]. The data [26] are coarse compared to present measurement and do not resolve the flow close to the hull, but still show good qualitative trends and agreement for FBKV $R$ and $\omega_{x}$. The key differences between the experiments include, in data [26] SDV and FBKV, are indistinguishable at $x=0.4, \mathrm{SDV}$ is stronger and persists until $x=0.6$, and $U$ is smaller/larger for SDV/FBKV. The solutions are qualitatively similar to the data, except for the fact that most solutions grossly overpredict the strength and extent of SDV. Both SDV and FBKV validations show similar trends, where the scatters and errors are small for $R$ and $U$, large for TKE, and very large for $Q$ and $\omega_{x}$. For SDV, $\bar{S}$ is close to $D$ for $R$ and TKE but overpredicts $Q$ and $\omega_{x}$ and underpredicts $U$, whereas for FBKV, $\bar{S}$ is close to $D$ for all variables.

Table 3 provides the streamwise averaged SoA assessment for the SDV and FBKV core variables. The trends are similar for both vortices. Both $U_{D}$ and $U_{\overline{S N}}$ have similar magnitudes and result in average $U_{V i} \sim 20 \% D$. For both SDV and FBKV, all solutions for $R$ and about one-fourth of the solutions for the other variables are validated. The scatter and errors $\sigma_{S} / \overline{|E|}$ are correlated such that they are very large for $Q(159 \% \bar{S} / 161 \% D)$, large for $\omega_{x}$ and TKE $(57 \% \bar{S} / 83 \% D$ and $50 \% \bar{S} / 45 \% D)$, and small for $R(3 \% \bar{S} / 4 \% D)$ and $U(4 \% \bar{S} / 9 \% D)$. The $Q$ predictions show large errors than $\omega_{x}$, as former is more or less square of the latter. For both the vortices, the errors are smallest for $\mathrm{S} 1$, and largest for S2. $U_{S O A}$ and $U_{S O A}$ intervals are very large about $70 \% D$ and $40 \% D$, respectively, and dominated by $P_{\left|E_{i}\right|}$. About three-fourth of the codes/solutions are $\mathrm{N}$-version validated, and only $R$ for both SDV and FBKV, and $Q$ and $U$ for FBKV are $\mathrm{N}$-version validated.

Figure 6 shows the $\mathrm{S} 1$ and S2 cross plane SDV vortex core validation at $x=0.2$ using zoomed in views, which highlights the detailed core structure of SDV. The predictions for both $x=0.2$ and 0.3 show similar trends. Experiments show that both SDV and FBKV have elliptical shape with major axes at angle of $20 \mathrm{deg}$ and $105 \mathrm{deg}$ to the center plane, respectively. The S1 and S2 contour plots are qualitatively similar to the experiments, except for S2 TKE, which only shows FBKV. Profile plots display deficiencies of both $\mathrm{S} 1$ and $\mathrm{S} 2$; however, the use of $y$ and $z$ cuts and the noncircular shape of SDV and FBKV accentuate the differences. Table 4 provides average validation uncertainty and errors. $U_{V i}$ is about $20 \% D$, whereas average errors for S1 and S2 are $35 \% D$ and $43 \% D$, respectively.

4.2 Static Drift $\boldsymbol{\beta}=\mathbf{2 0}$ deg. Figure 7 shows the vortical structures in the flow, similar to Fig. 3 for $\beta=0 \mathrm{deg}$. The flow is asymmetric with respect to the center plane and the effects of the static drift angle dominate the flow. The crossflow from windward (WW) to leeward (LW) sides generates two primary vortices with counter clockwise rotation due to the sonar dome and WW bilge keel. The experimental data in Fig. 7(a) clearly display the sonar dome (SDTV) and bilge keel (BKTV) tip vortices whose strength and progression are different from those shown for SDV and 


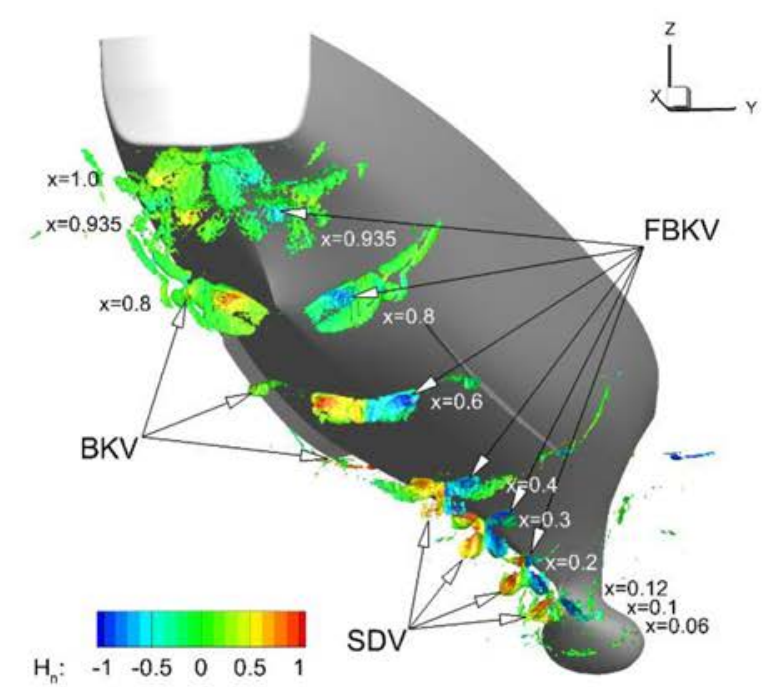

(a)
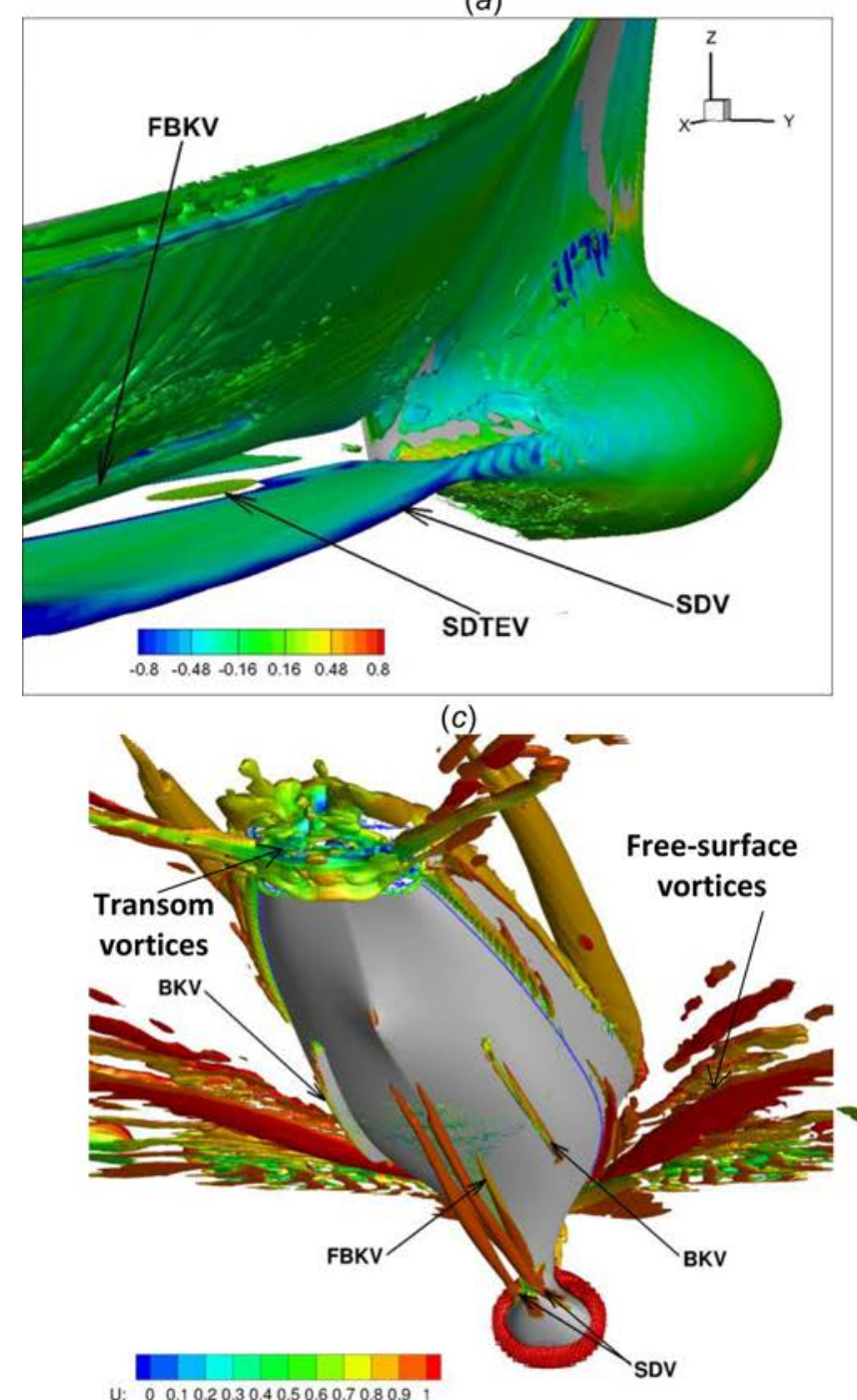

(e)

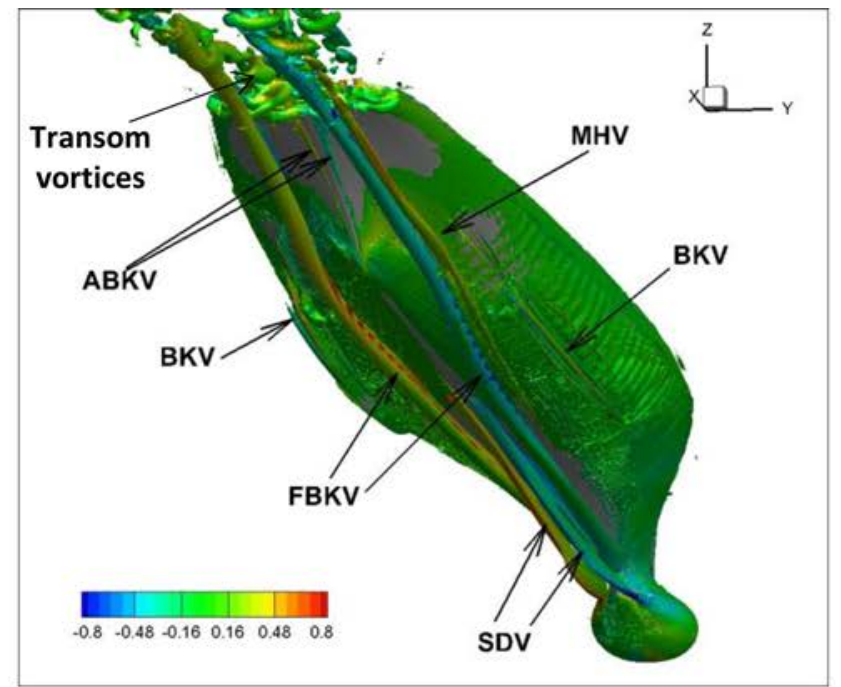

(b)

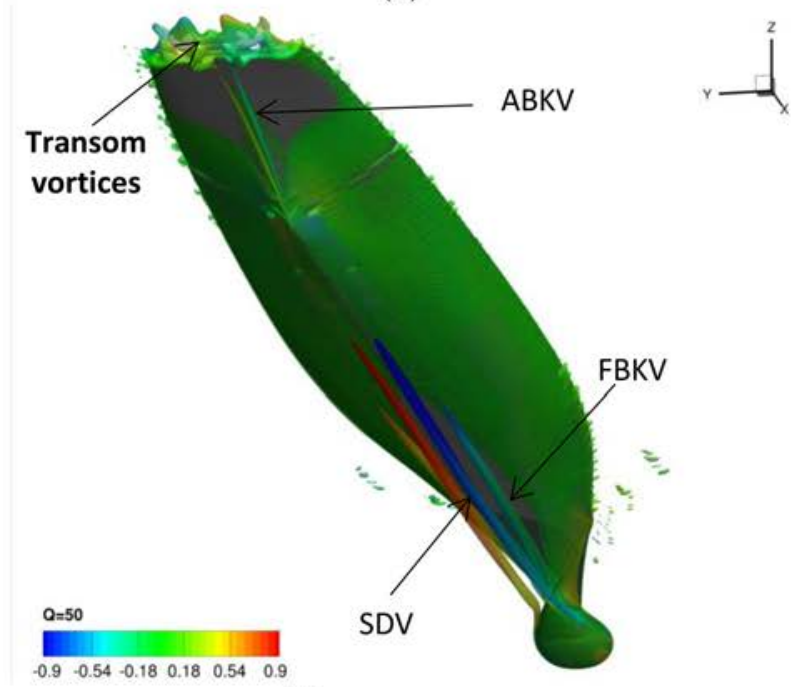

(d)

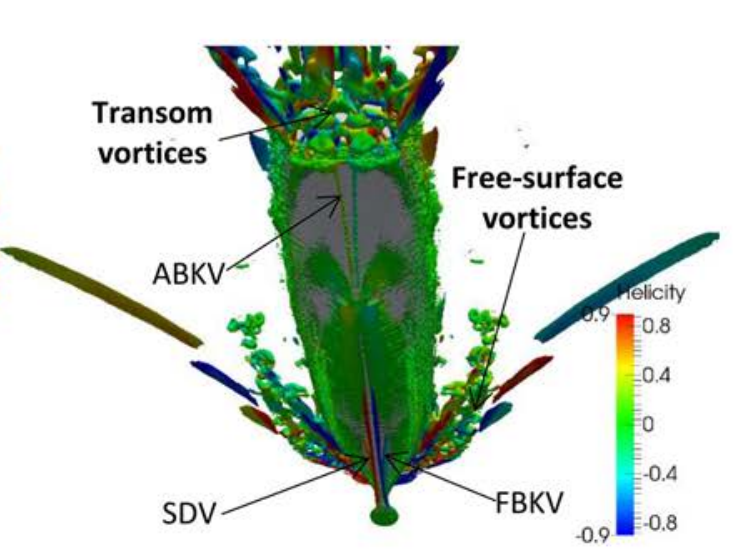

$(f)$

Fig. 3 Iso-surfaces of $Q=50$ colored by relative helicity for $\beta=0$ deg. Subplots show results obtained from (a) experiment, (b) S2, (c) S2 (zoomed in view), (d) S3, (e) S4, and (f) S6. S4 and S5 also reported SDTEV, which are not visible in the plots.

FBKV. SDTV is first observed at $x=0.1$ and BKTV at $x=0.6$. Additional secondary vortices are also observed: counter-rotating WW- and LW-FBKV with same rotation directions as PS and SS for $\beta=0 \mathrm{deg}$; clockwise rotation $\mathrm{ABKV}$; WW- and LW-free- surface vortices (FSV) due to wave breaking with clockwise and counter clockwise rotation on the LW and WW. WW-FBKV is first observed at $x=0.2$, and LW-FBKV at $x=0.3$. ABKV is first observed at $x=0.8$. LW-FSV is observed at $x=0.12$, and WW- 


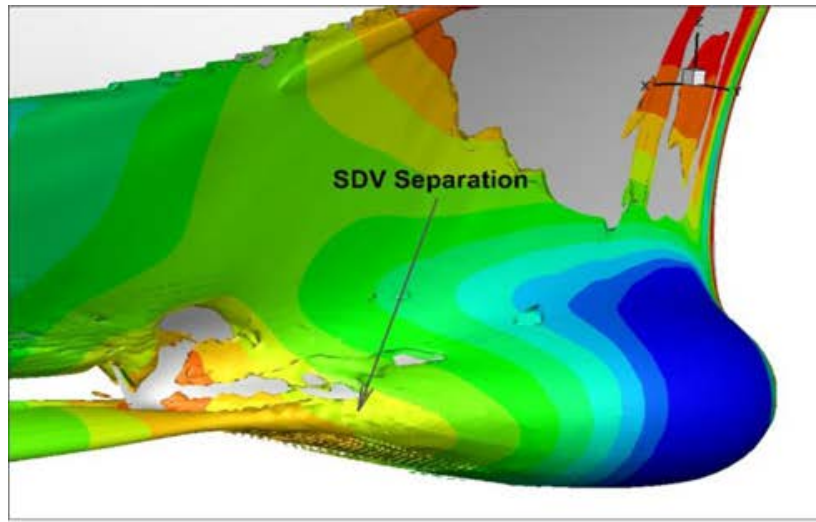

(a)

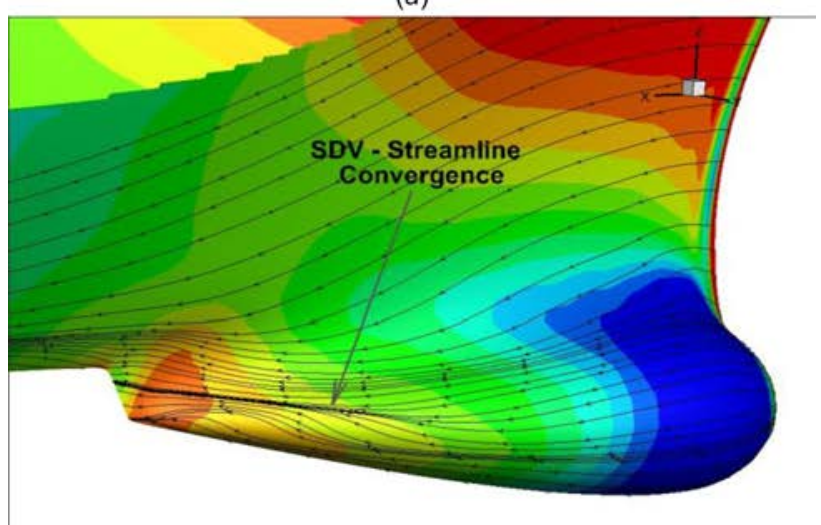

(b)

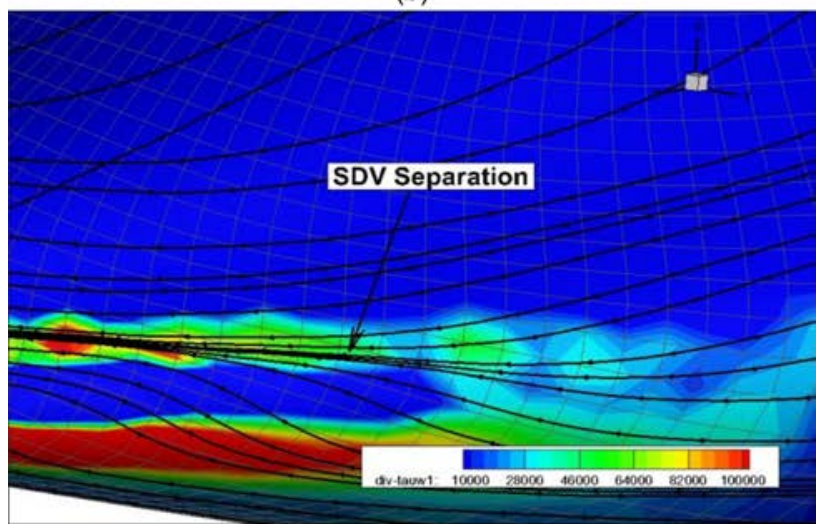

(c)

Fig. 4 Zoomed-in view of sonar dome shows SDV flow separation predicted by $S 1$ for $\beta=0$ deg. (a) Iso-surface of $Q=100$ shows SDV separation, and $(b)$ surface streamline shows onset of SDV. Both plots (a) and (b) are colored using pressure. (c) Contours of wall shear stress distribution of $\operatorname{div}\left(\tau_{w}\right)$ along with surface streamline.

FSV at $x=0.2$. The $\beta=10 \mathrm{deg}$ data showed similar vortices, except the SDTV strength reduced, both BKTV and ABKV strengths increased, TKE decreased for all the vortex cores, and FSVs were absent. Additionally, SDTV and BKTV display circular cross plane streamlines with normalized cross plane profiles showing Gaussian/bell-shaped similarity.

As shown in Figs. 7(b)-7(d), the S2, S4, and S6 results show all the primary vortices observed in the experiments, and some additional secondary vortices. Solutions have some similarities and some dissimilarities for the prediction of secondary vortices. Experiments could not confirm the secondary vortices. S2 exhibits helical mode instability for SDTV and BKTV. S4 and S6 did not investigate the instantaneous solution to identify such instability.
Onset and separation analysis using time-averaged $\mathrm{S} 1, \mathrm{~S} 2$, and S3 indicate that SDTV, LW/WW-FBKV, BKTV/BKV, MHV, and ABKV are all open-type/crossflow separations. SDTV onset is from the LW sonar dome surface at $x=0.088$, and shows helical mode instability starting at $x=0.11$. Both LW- and WWFBKV onset are at $x=0.2$, and progress on the LW side. BKTV onset is from the WW bilge keel at $x=0.4$, and shows helical mode instability right after onset. BKV shows pairs of counterrotating vortices originating from the $\mathrm{LW}$ bilge keel leading edge at $x=0.4$. The anticlockwise and clockwise vortices originate from the outer and inner surfaces of the bilge keel, respectively. MHV onset is from the LW mid-girth at $x=0.6$ between the bilge keel and the keel. ABKV shows pairs of counter rotating vortices from the keel that onset at $x=0.8$ where the after-body hull curvature starts.

Predictions show closed bubble-type separation on the LW bow (BSB). The separation of the vortex is analyzed using S2 in Fig. 8, which shows $Q=1000$ iso-surfaces and surface streamlines on the LW bow, and a $z=-0.04$ cut across the bubble. BSB originates from a sink node $\left(N_{P}\right)$ at the leading edge, is encased by a saddle point $\left(S_{P}\right)$, and terminates at two focus points $\left(F_{P}\right)$. This satisfies the topological rule expected for a close separation over an isolated body [29]

$$
\sum\left(N_{P}+F_{P}\right)-\sum S_{P}=2
$$

The separation bubble sheds a vortex SBV, due to merger of multiple separations from the bubble. The vortex originates from a saddle point, does not terminate on the surface, and shows diverging streamlines downstream. The vortex has similarities with open-closed separation at onset, but shows differences as downstream flow neither shows converging streamlines nor terminates at a limit cycle.

On the WW bow, a sheet of fluid climbs vertically up as the flow encounters stagnation point on the hull. In addition, the high pressure on the hull further imparts an outward momentum on fluid, generating a plunger, which falls back on the free-surface due to gravity. The upward and downward flows in the plunger and reconnecting sheets, respectively, generate a counterclockwise axial vorticity in the flow. The plunger/reconnection creates an air pocket that remains exposed to ambient pressure and closes further downstream, leaving wake of axial vorticity in the flow and free-surface scar at reconnection. Flow also shows secondary and tertiary plunger/reconnection, similar to the primary plunger/reconnection, but with decreasing plunger height and axial vorticity strength. Wilson et al. [30] reported a similar breaking wave pattern for Athena bow waves for higher $\mathrm{Fr}=0.43$ and 0.62 . The primary plunger/reconnection results in a vortex, which is identified as WW-FSV. Topological analysis shows that the vortex separates from a source node close to the leading edge and attaches at a saddle point on the free-surface and another on the hull. The vortex satisfies the topological rule expected for a close separation formed over a body intersecting a wall or freesurface [31]

$$
\left\{\sum\left(N_{P}+F_{P}\right)-\sum S_{P}\right\}_{F S+B}=-1
$$

where $F S$ is the free-surface and $B$ is the hull body. WW-FSV involves multiple plunger/reconnection, thus exhibiting spilling breaking.

The LW bow lies in the shadow region of the flow, and thus has overall lower surface pressure resulting in lower wave elevation. The wave elevation is high close to the leading edge; thus, the flow encounters a sharp elevation drop, which forms a threedimensional plunger/reconnection. The plunger/reconnection leaves a wake of clockwise rotating vortex LW-FSV similar to WW-FSV. However, unlike WW-FSV, it creates air-bubble pockets, has three times higher plunger amplitude, and does not show secondary plunger/reconnection. LW-FSV originates from a 

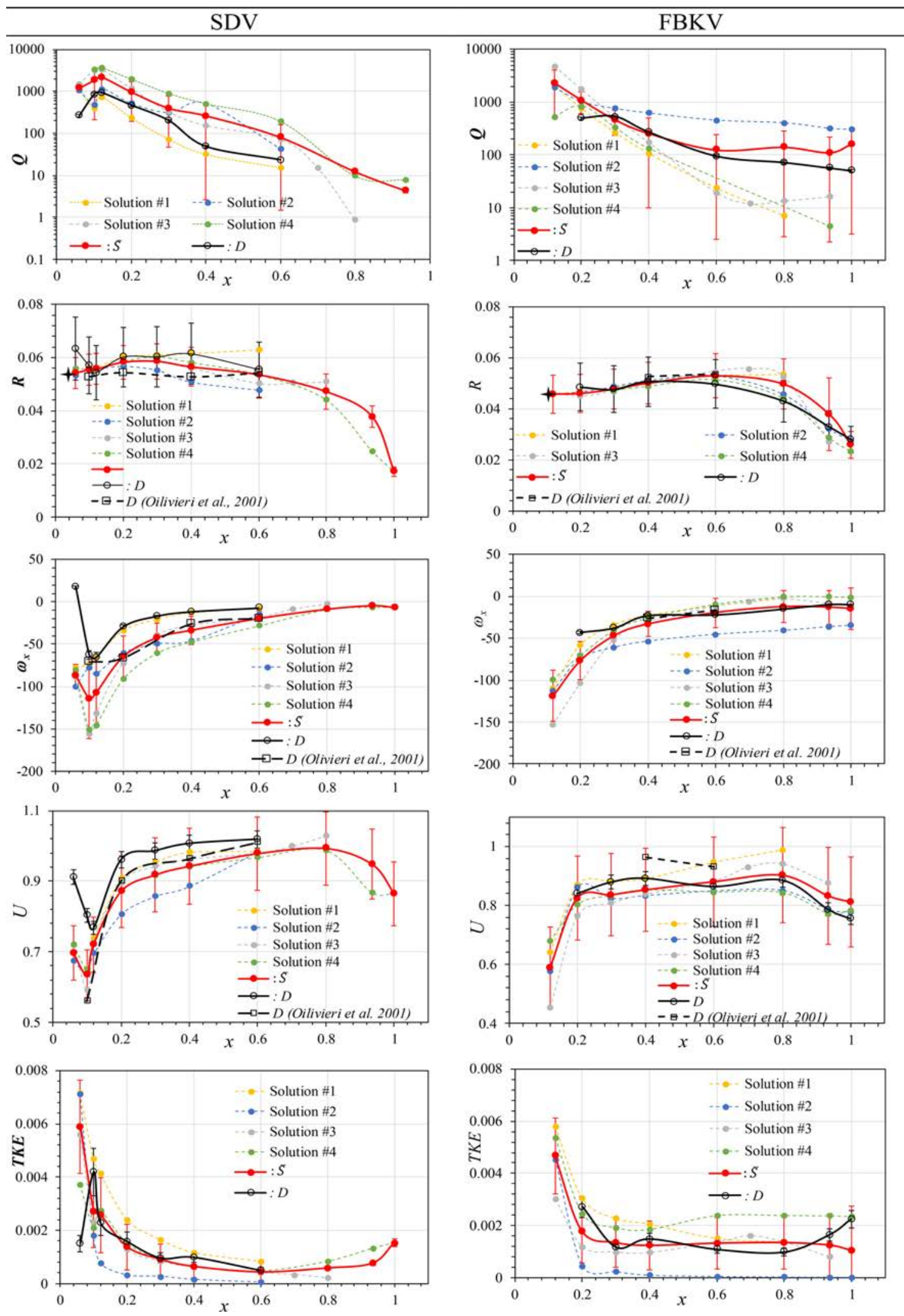

Fig. 5 Axial variation of flow variables in SDV (left column) and FBKV (right column) cores obtained using S1 through S4, and mean code with $P_{\text {b }}$ bars are compared with experimental data with $U_{D}$ bars for $\beta=0$ deg. The SDV onset at $x=0.0635$ and FBKV onset at $x=0.107$ are shown in the radial location $(R)$ subplot using the star symbol.

saddle point underneath the free-surface, does not terminate on the surface, and shows converging streamlines downstream. Thus, its separation is open-closed type separation. LW-FSV has single plunger/reconnection, thus exhibiting plunging breaking.
Figure 9 is similar to Fig. 5, but shows vortex core variables for SDTV and BKTV and has a much larger scale. The experiments indicate that the characteristics of SDTV and BKTV are different from SDV and FBKV. SDTV and BKTV progress on the LW side 

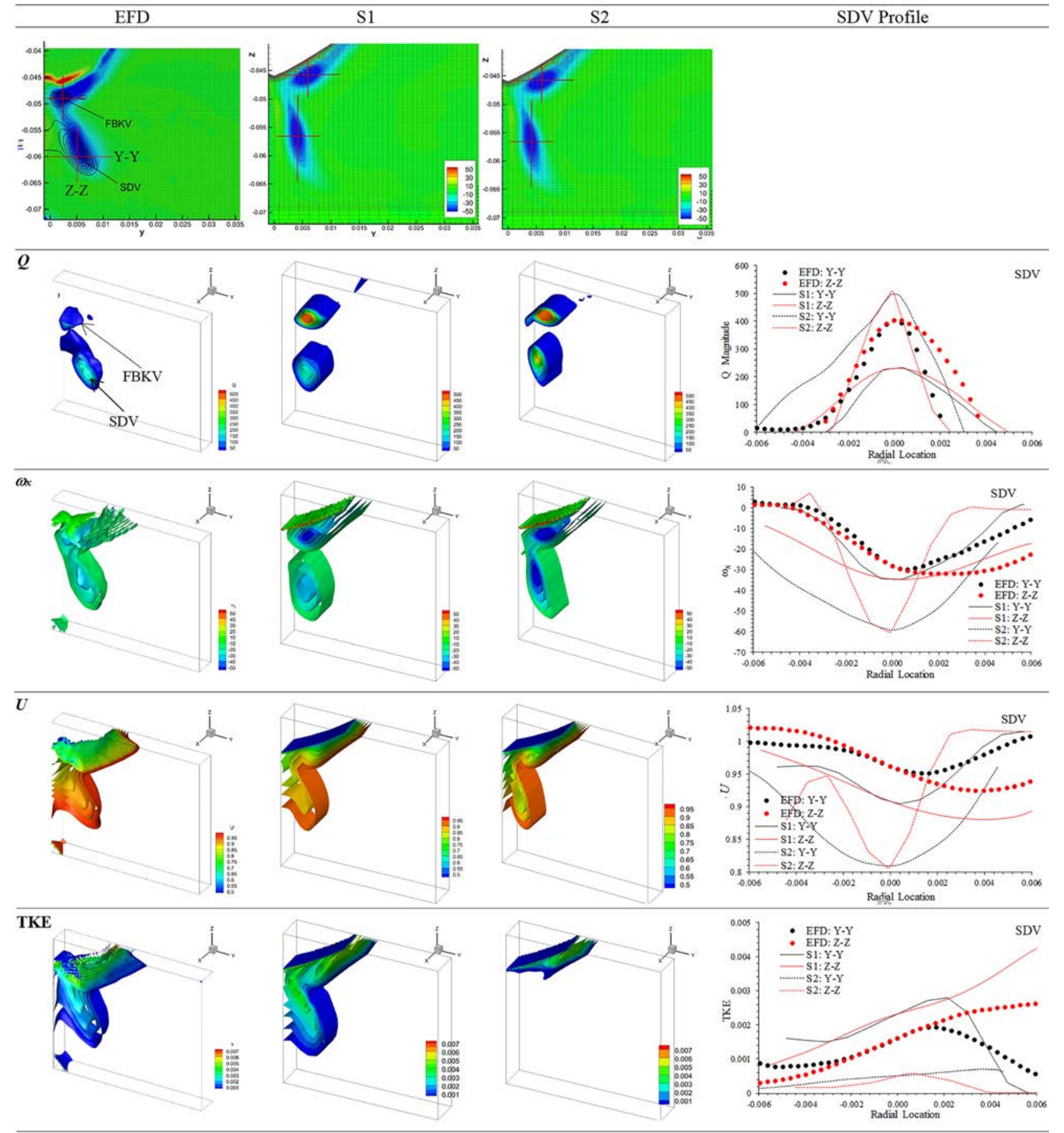

Fig. 6 Cross plane vortex core predictions for $\beta=0$ deg at $x=0.2$. (Top row) Contour of axial vorticity, contour lines of $Q$, grid lines, and cross-plane analysis Y-Y and Z-Z lines. Volume plots showing 3D structures of the SDV and FBKV flow variables obtained the experimental data (left), S1 (2nd column) and S2 (3rd column). Subplots on the (right) are compared with $y-y$ and $z-z$ profiles of the flow variables in the SDV core predicted by $S 1$ and $S 2$ with the experimental data. For $y$-y line, the -ve radial location is toward the center plane. For $z-z$ line, the -ve radial location is away from the hull.

at an angle $10 \mathrm{deg}$ with respect to the keel, whereas SDV and FBKV progress along the hull and interact with the boundary layer. $U$ shows steady increase in velocity deficit during progression, whereas $\beta=0$ deg vortices show a recovery in the deficit. Vortices for $\beta=20$ deg show two orders of magnitude higher $Q$ and almost half of the decay rate, an order of magnitude higher $\omega_{x}$ and four times faster decay compared to the $\beta=0 \mathrm{deg}$ vortices. The vortices also show an increase in $Q$ and TKE during early progression, followed by a sharp change at $x=0.12$. These characteristics are consistent with vortex breakdown and helical mode instability. After the breakdown, TKE shows a decrease early on and constant value further downstream. The CFD trends roughly follow the experiments, except for TKE. $R$ shows close agreement. $Q, \omega_{x}$, and $U$ are underpredicted and overpredicted for SDTV and BKTV, respectively. For both vortices, TKE shows significant underprediction. Both S1 and S2 show low pressure initially, which recovers very rapidly during early progression, which is consistent with vortex breakdown.

Table 3 includes SoA assessment for SDTV and BKTV. The trends are similar for both vortices and similar to $\beta=0$ deg results. 


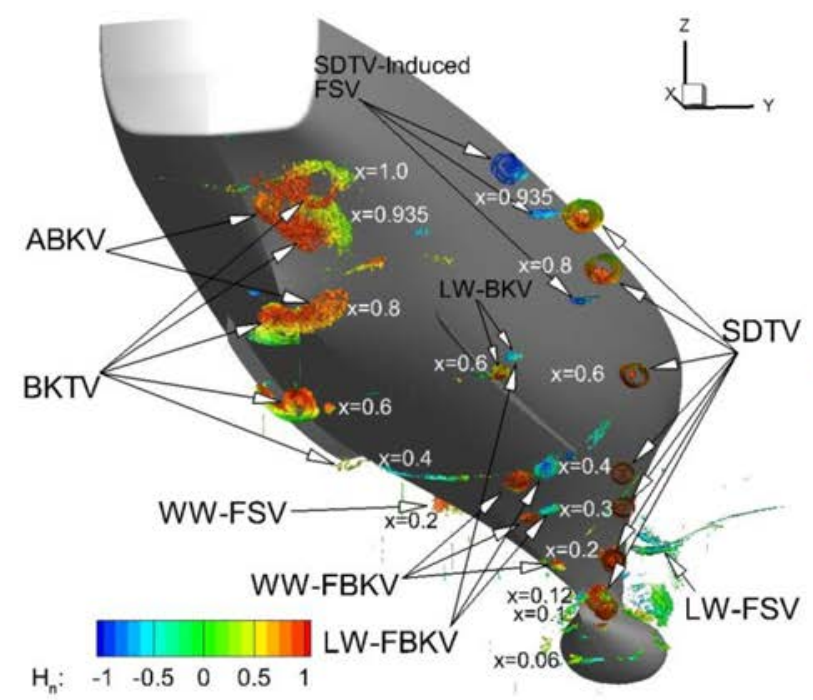

(a)

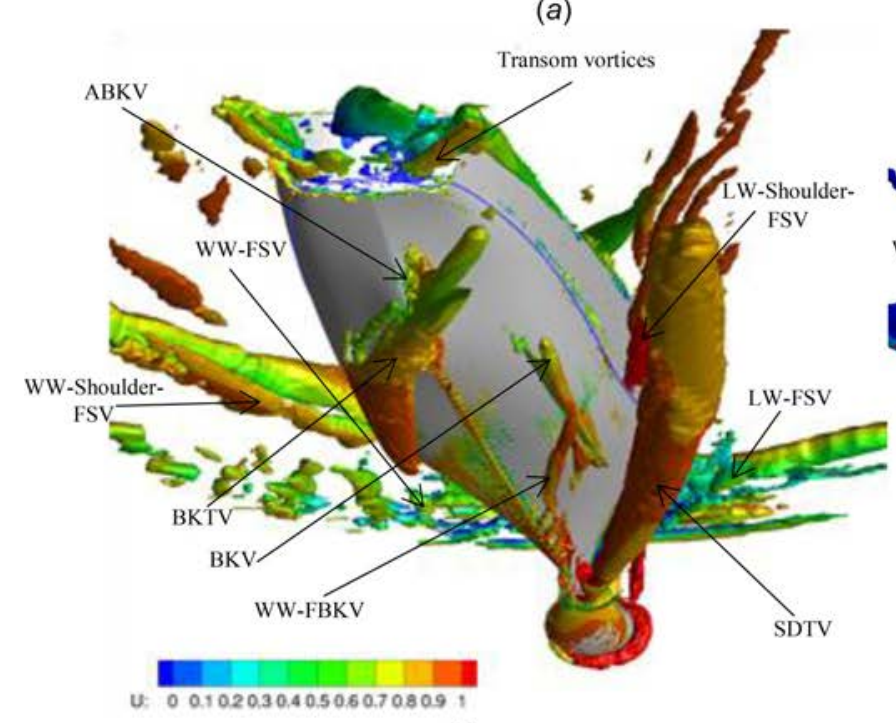

(c)

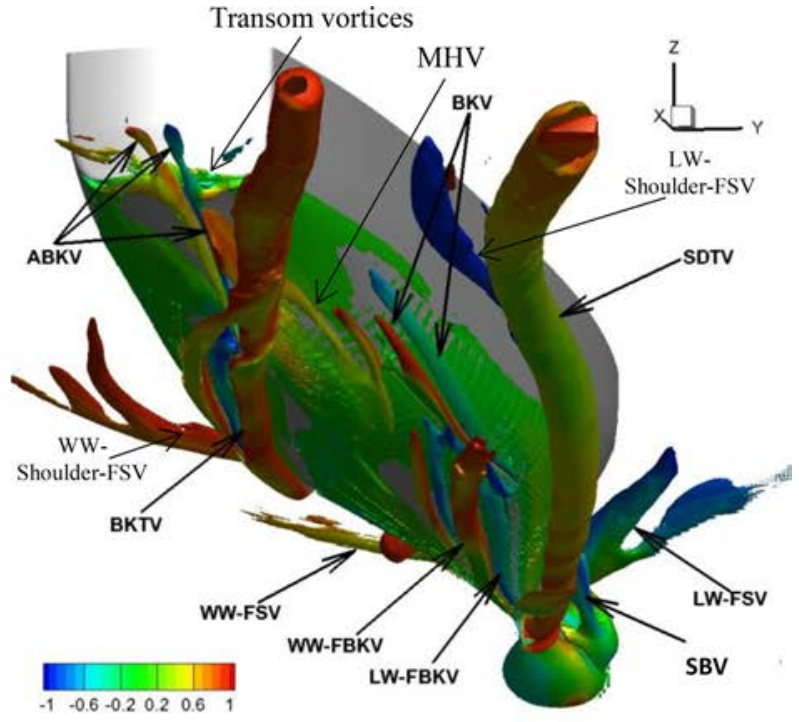

(b)

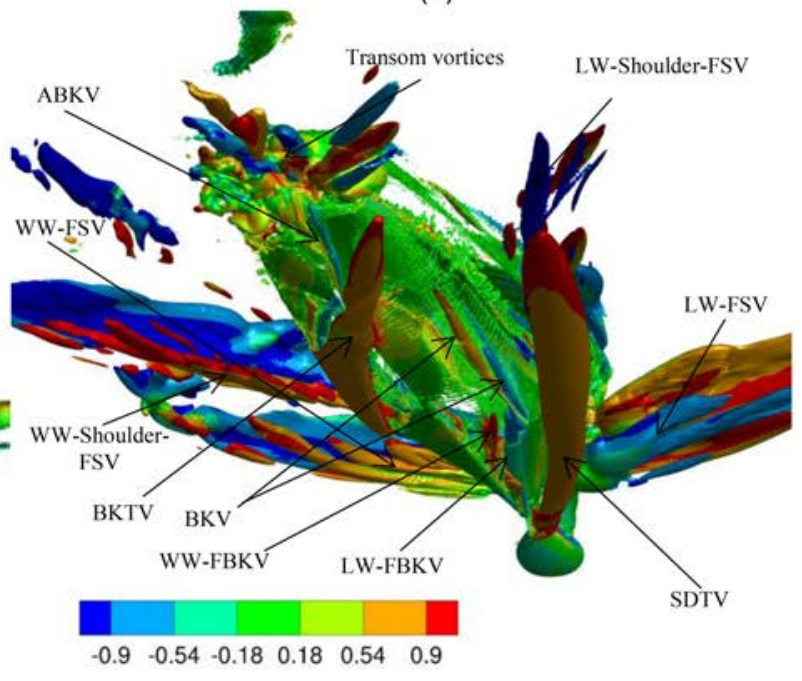

(d)

Fig. 7 Iso-surfaces of $Q=100$ colored by relative helicity for $\beta=20$ deg. Subplots show results obtained using: (a) experiment, (b) S2, (c) S4, and (d) S6.

$U_{\overline{S N}}$ is about six times larger than $U_{D}$ with average $U_{V i}$ about $20 \% D$. For both SDTV and BKTV, around three-fourth of $R$ and $U$ solutions are validated, whereas limited solutions are validated for other variables. The scatter and errors $\sigma_{S} /|E|$ are correlated such that they are very large for $Q(75 \% \bar{S} / 126 \% D)$, large for $\omega_{x}$ $(25 \% \bar{S} / 47 \% D)$ and TKE $(30 \% \bar{S} / 70 \% D)$, and small for $R(4 \% \bar{S} /$ $5 \% D)$ and $U(8 \% \bar{S} / 7 \% D)$. On an average $\mathrm{S} 1, \mathrm{~S} 2$, and $\mathrm{S} 4$ show similar error levels, and $\mathrm{S} 3$ shows the largest error. $U_{S O A}$ and $U_{S O A}$ intervals are large about 40 and $30 \% \mathrm{D}$, respectively. $P_{\left|E_{i}\right|}$ is the dominant uncertainty component for BKTV $Q, \omega_{x}$ and TKE, whereas for the rest, $U_{V i}$ and $P_{\left|E_{i}\right|}$ are comparable. For both SDTV and BKTV, around three-fourth of the $R$ and $U$ solutions are validated. Around one-fourth and half of $Q, \omega_{x}$, and TKE solutions for SDTV and BKTV are validated, respectively. Mean code for SDTV $R$ and BKTV $U$ is N-version validated. The primary difference between SDTV and BKTV predictions is the latter shows three times large error for $Q$. The primary difference between the errors for $\beta=0 \mathrm{deg}$ and $20 \mathrm{deg}$ predictions is the latter shows two times large error for TKE.

Figure 10 is similar to Fig. 6, but for SDTV at $x=0.2$. Experiments show that SDTV is roughly circular at $x=0.2$ and 0.4 , but somewhat elliptical at $x=0.8$ with major/minor axes in the $z / y$ directions. $Q, \omega_{x}$, and TKE contours show asymmetric core with crescent shaped region with largest values. $U$ contours have dipole and circular shape at $x=0.2 / 0.4$ and 0.8 , respectively. Profiles exhibit trends using $y$ and $z$ cuts, including core asymmetry and twin peaks especially for $x=0.4$. S1 and especially S2 contour plots are similar to the experiments. Here again, the profile plots accentuate the differences. BKTV results at $x=0.6$ show the vortex under the hull at about midgirth with tail extending to the bilge keel, where another vortex is forming. BKTV has a circular shape, whereas the secondary vortex has elliptical shape. Table 4 shows that $U_{V i}$ is about $20 \% D$, whereas average errors for $\mathrm{S} 1$ and $\mathrm{S} 2$ are $58 \% \mathrm{D}$ and $35 \% \mathrm{D}$, respectively.

\section{Effects of Grid Resolution and Turbulence model}

5.1 Straight Ahead $\boldsymbol{\beta}=\mathbf{0}$ deg. S1 resistance, wave elevation, and vortex core predictions improved when grid was refined from $10 \mathrm{M}$ to $48 \mathrm{M}$, but further refinement does not show significant improvements. S2 resistance and wave elevation predictions showed similar trends with grid refinement. However, the vortex core prediction errors increased with refinement because of the increase in the overprediction of vortex strengths. S3 resistance predictions improved with grid refinement from $1.3 \mathrm{M}$ to $10.2 \mathrm{M}$ cells, but further refinement does not significant improvement. S4 vortex resolution improved with grid refinement, but the 


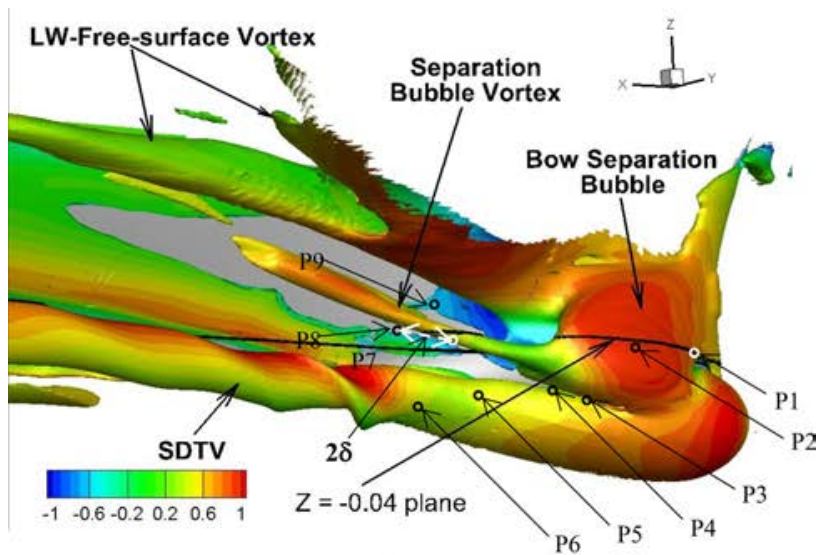

(a)

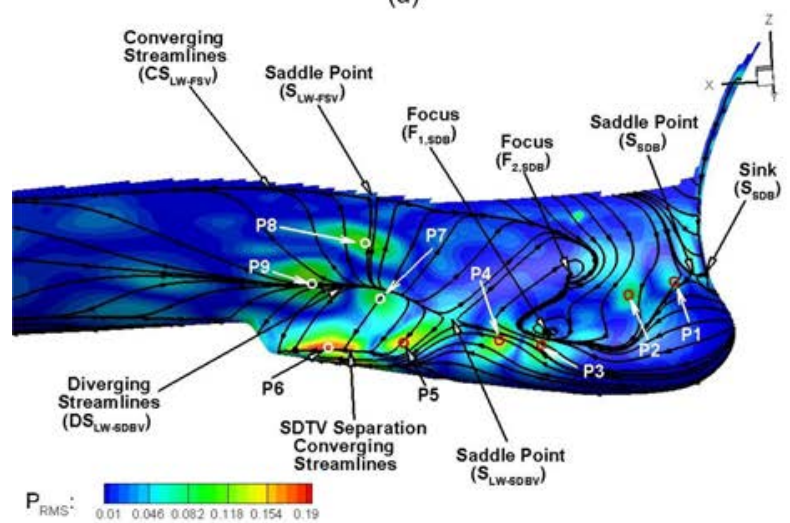

(b)
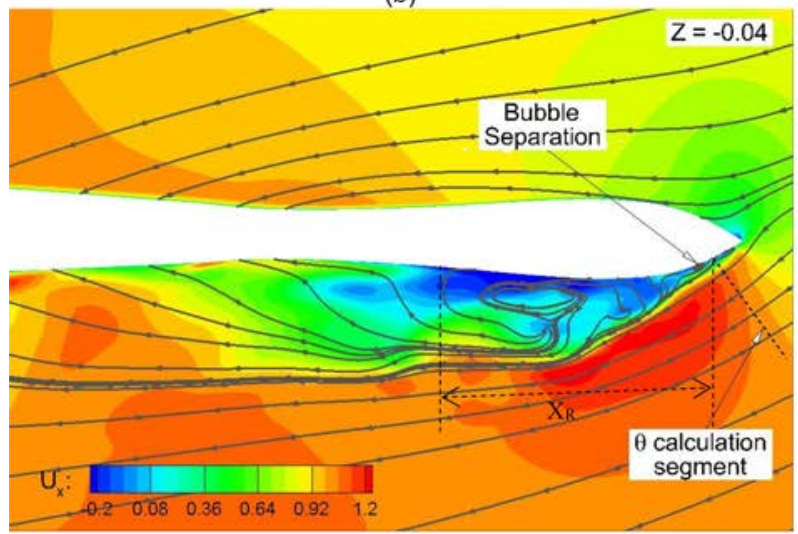

(c)

Fig. 8 S2 predictions on leeward sonar dome surface for $\beta=20$ deg showing: (a) mean flow iso-surfaces of $Q=1000$ colored by relative helicity. The points $\mathrm{P} 1$ to $\mathrm{P} 9$ correspond to the probe points on the hull for the FFT analysis. (b) Mean flow surface streamlines and root-mean-square of the surface pressure $\left(P_{\mathrm{RMS}}\right)$. The streamline patterns identified by subscript LW-FSV, BSB, and SBV correspond to leeward free-surface vortex, sonar dome separation bubble, and leeward sonar dome bubble vortex, respectively. (c) Instantaneous flow predictions on the leeward sonar dome surface at $z=-0.04$ plane showing shear layer instability associated with sonar dome bubble separation.

resistance prediction errors increased from $3.8 \%$ on $22 \mathrm{M}$ grid to $6.2 \%$ on $81 \mathrm{M}$ grid. S5 also reported an increase in resistance prediction errors from $2.2 \%$ to $5.7 \%$ when the grid was refined from $9 \mathrm{M}$ to $51 \mathrm{M}$ cells. The relatively large errors in S4 and S5 on fine grids were attributed to poor grid resolution of the free-surface close to the sonar dome region. S6 refined the grid in the area close to the hull, free-surface and bilge keels, and reported improvement in the resistance predictions with grid refinement; however, the grid refinement range was rather small. Overall, considering the individual grid study and different solutions with varying turbulence and near-wall models, and blockage effects, one can conclude that grids with $10 \mathrm{M}$ cells provide sufficiently accurate resistance and free-surface predictions. S3 indicates that the effect of blockage on resistance is within the validation uncertainty interval of about 5\%. S6 indicates that wall-functions perform as well as near-wall models when wall grid resolution $\mathrm{y}^{+}$is as close as possible to the lower log-law ( 30).

It is essential to use sufficiently fine grid resolution at the sonar dome to capture the onset of the vortical structures accurately, in particular FBKV and SDTEV. However, for the progression of vortices, numerical dissipation is the largest influencing factor. Thus, both grid resolution and turbulence modeling are important. Egolf et al. [32] concluded that a minimum of 14 grid points across the vortex core and a theoretically fifth-order accurate scheme are required for "minimal vortex diffusion over a long distance." It is expected that solutions using second-order schemes (as used by most submissions) would require a significantly large number of cells in the vortex core to achieve similar diffusion. This means that the grid should be adapted to the trajectories of the vortices to avoid any excessive dissipation of the vorticity. S1/ S2 achieves grid refinement by using overset methodology, where the coarse grid solutions guide the placement of the refinement blocks. S3 grid study used geometrically similar grids obtained by refining grids blocks in all directions simultaneously. S6 used locally embedded blocks of fine grids based on coarse grid solutions. S4 used automatic grid refinement strategy using local Hessian of pressure. Considering both the grid resolution and numerical accuracy, none of the simulations meets the strict requirements of [32]. Only S2 that uses fourth-order scheme and has 12 cells in the vortex cores is close to satisfying them.

Comparing turbulence models for the vortex progression, S2 provides better resolution of the vortical structures over the foreand midbody than S1, e.g., they capture the closed streamline at $x=0.6$ as shown in Fig. 11. However, they predict very low resolved turbulence levels. An additional simulation performed using ISIS-CFD DES also showed very low resolved TKE levels similar to S2. S3 compared variant of isotropic $k-\omega$ SST models, and reported that SST2003 [23] performs slightly better than other variants. S4 predictions showed that anisotropic URANS turbulence model performs better than the isotropic model. Overall, close to the vortex onset region, both DES and URANS turbulence models show similar results. This is primarily because DES operates in URANS mode in these regions. In the near wake of the sonar dome, the flow appears to be mostly pressure-driven. The vortices progress in the flow along the hull, and the numerical dissipation is the largest influencing factor. This means that the grid should be adapted to the trajectories of the vortices to avoid excessive dissipation of the vorticity. This advocates for local mesh adaptation that can automatically refine grids in the regions with flow characteristics of interest. However, the effectiveness of mesh adaptation method in tied to the turbulence modeling, as isotropic and anisotropic URANS and DES models show significant differences in vortex progression. The following general conclusions are drawn:

(1) URANS models predict too weak longitudinal vortices that dissipate more quickly compared to the data. However, the amount of TKE is predicted reasonably well up to $x=0.6$. The turbulence production is proportion to the rate of strain; thus, weak vortices suggest lower TKE production. Thus, a reasonable TKE prediction suggests overprediction of transport/diffusion of turbulence. From $x=0.6$ to the stern, the predicted TKE is too high in the region between the vortex core and the hull, which is most likely the cause of excessive decay of the vortices.

(2) Anisotropic URANS models are marginally better than the isotropic counterparts, as the flow involves significantly large effects of streamline curvature in and around the 

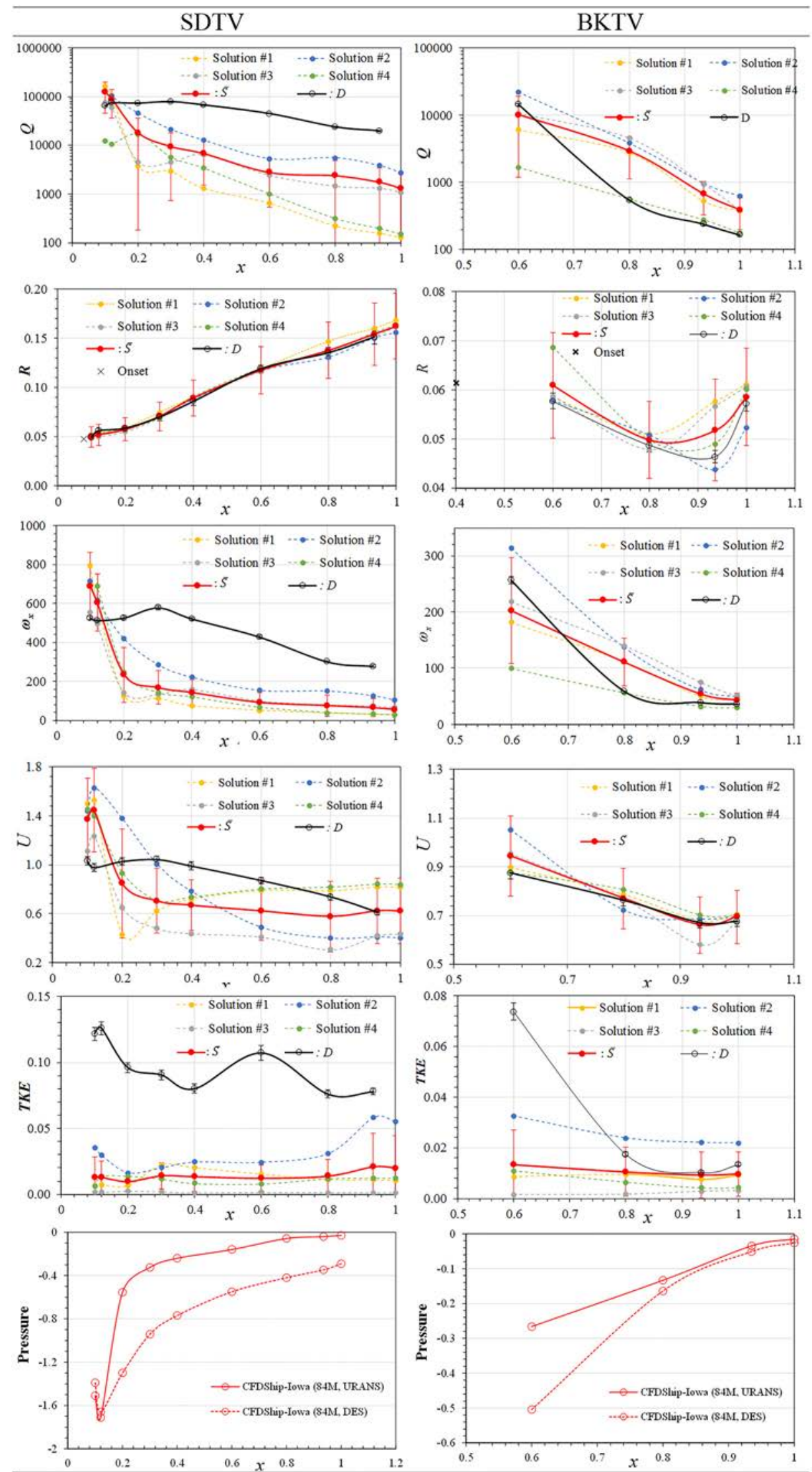

Fig. 9 Axial variation of flow variables in SDTV (left column) and BKTV (right column) cores obtained using $S 1$ through $S 4$, and mean code with $P$ bars is compared with experimental data with $U_{D}$ bars for $\beta=20$ deg. The SDTV onset at $x=0.077$ and BKTV onset at $x=0.4$ are shown in radial location $(R)$ subplot. 


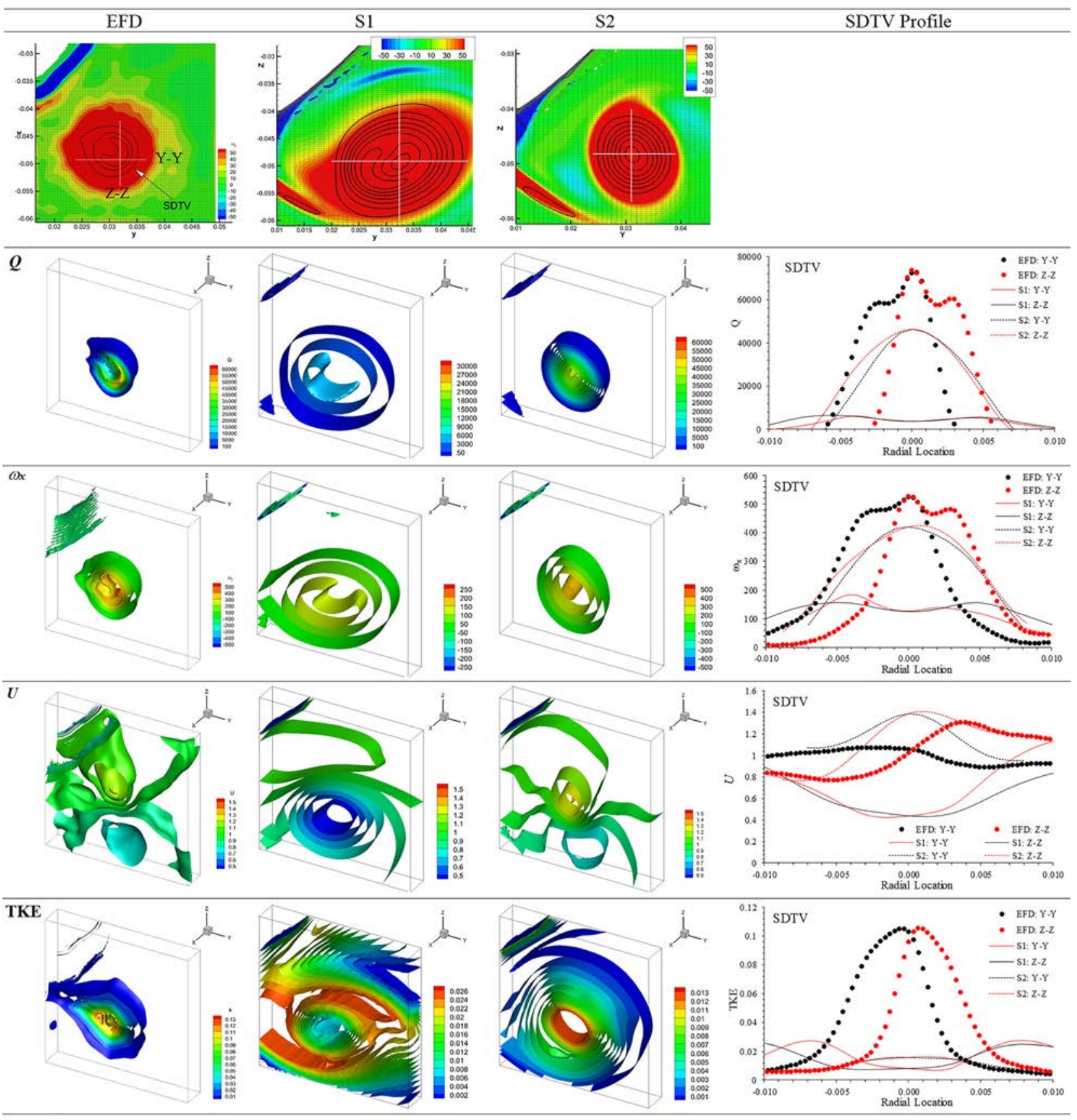

Fig. 10 Cross plane vortex core prediction for SDTV for $\beta=20 \mathrm{deg}$ at $x=\mathbf{0 . 2}$. (Top row) Contour of axial vorticity, grid, and cross-plane analysis $Y Y$ and $Z Z$ lines. Volume plots showing 3D structures of the SDTV flow variables obtained the experimental data (left), S1 (2nd column), and S2 (3rd column). Subplots on the (right) are compared with $y-y$ and $z-z$ profiles of the flow variables in the SDTV core predicted by $S 1$ and $S 2$ with the experimental data. For $y-y$ line, the -ve radial location is toward the center plane. For $z-z$ line, the -ve radial location is away from the hull.

vortex cores. This suggests that the available anisotropic models are unable to reproduce this physics accurately.

(3) DES looks promising insofar as they predict more persistent longitudinal vortices than URANS, which are primarily due to significant underprediction of TKE resulting in a quasi-laminar core. The flow laminarization is caused by the modeled stress depletion issue in DES, and cannot be resolved by grid refinement [33].

5.2 Static Drift 20 deg. S1 performed grid study using $10 \mathrm{M}$, $48 \mathrm{M}$, and $84 \times 10^{6}$ grids, which have similar resolution as $\beta=0 \mathrm{deg}$ and the overset blocks were adjusted according to the vortex progression directions. S2 grid study included the above three grids and an additional $250 \mathrm{M}$ grid. The largest grid was not properly adapted. Thus, the vortex core resolution was larger than those of $84 \mathrm{M}$ grid. For S1, forces, and moment, wave elevation and vortex core predictions did not improve when grid was refined from $48 \mathrm{M}$ to $84 \mathrm{M}$ grid, similar to $\beta=0 \mathrm{deg}$. For $\mathrm{S} 2$, forces, moment and wave elevation showed a similar trend. However, vortex core predictions improved with grid refinement and further improvements are possible on finer grids. S3 reported improvements in forces and moment predictions with grid refinement from 1.3 $\mathrm{M}$ to $17 \times \mathrm{M}$ cells, but did not show significant improvement upon further refinement to $76 \mathrm{M}$ cells. S6 reported $7 \%$ improvement in forces and moment for grid refinement from 18 

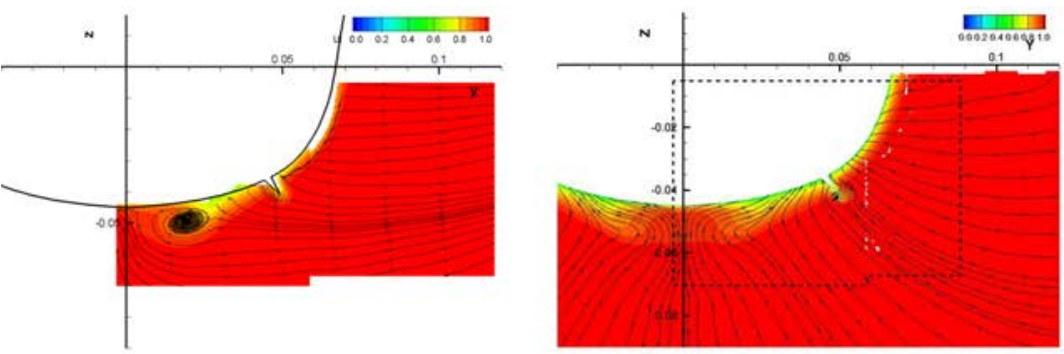

(a)

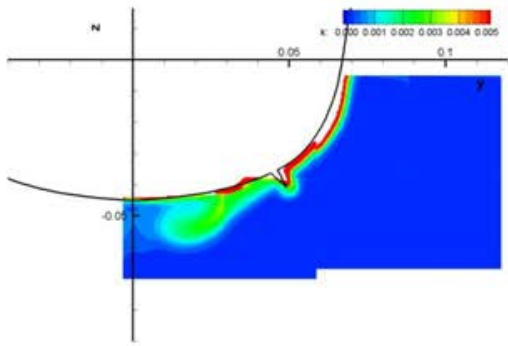

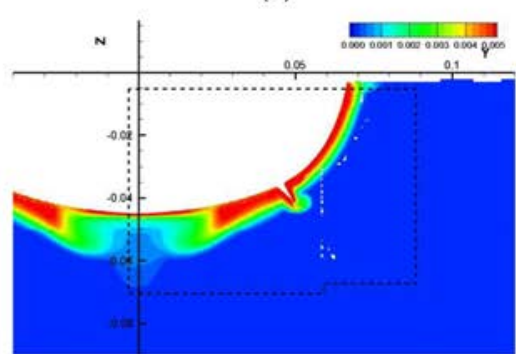

(b)
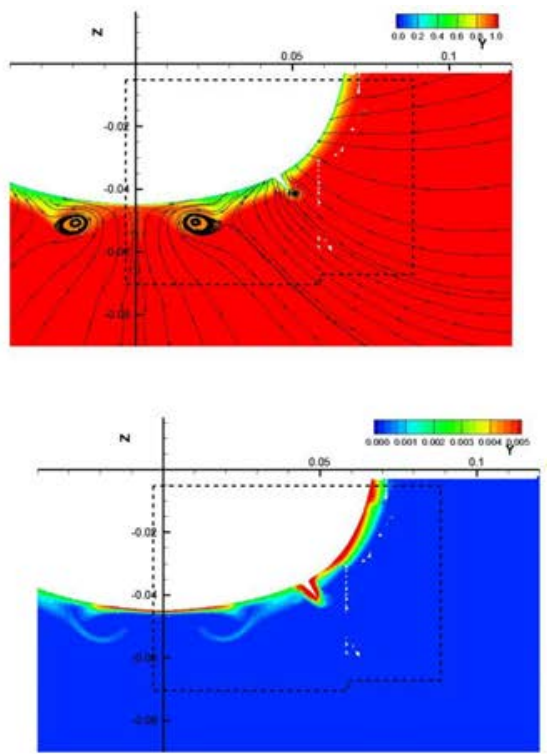

$\mathrm{M}$ to $68 \mathrm{M}$ points. S7 also reported improvement in forces and moment with grid refinement near the hull.

Overall, the contributions involved different grid sizes, wall models, and tow-tank sidewall blockage effects. Forces and moment predictions do not show a consistent trend with grid resolution, e.g., both S6 and S8 show similar large errors even though they use order of magnitude different grid resolutions. Both the near-wall and WF models show similar predictions of forces; however, the former is more accurate than the latter for yaw moment. S3 and S6 agree that the blockage does not significantly affect $X$ and $N$ predictions, however disagree regarding their effect on $Y$. Further investigation is required to evaluate the effect of grid sizes, wall models, and blockage on forces and moment.

For the wave elevation predictions, the errors were mostly larger than those for $\beta=0 \mathrm{deg}$, which were attributed to the coarse far-wake grid resolution in S1, S2, and S4, and to wall interference in S3 and S6. Similar to $\beta=0 \mathrm{deg}$, the effects of turbulence model and wall models were not discernable. VoF predicted higher wave elevation and more waves breaking than level-set near the bow, but showed similar results away from the hull.

The flow for $\beta=20 \mathrm{deg}$ is far more complex than that for $\beta=0 \mathrm{deg}$ and shows multiple vortices emanating along the hull, most notably from the sonar dome and bilge keels. Moreover, the free-surface deformation is quite large leading in unsteady breaking waves ensuing bubbly mixture of air and water and associated vortical structures near the free-surface. Further, contrary to the $\beta=0 \mathrm{deg}$, most of the vortices progress far from the hull after their separation. Thus, the evolution of the mean flow and turbulence characteristics along the vortex cores are quite different. Considering both the grid resolution and numerical accuracy, only S2 is close to satisfying the minimal vortex diffusion requirements of Ref. [32], as it uses fourth-order scheme and resolves the SDTV and BKTV cores using 20 and 15 cells, respectively.

$\mathrm{S} 1$ and S2 vortex core predictions show that DES provides better prediction of vortex strength and TKE than URANS. S4 results show that anisotropic URANS predicts better vortical structures than isotropic URANS, but they do no show significant differences for planar and volume plots. Comparison between S4 and S5 results shows that $10 \mathrm{M}$-AGR grid shows better prediction than nonadapted $27 \mathrm{M}$ grid especially for SDTV vortex strength and TKE.
Experiments show high level of TKE in the SDTV core from $x=0.4$ down to the last station. The persistently high level of TKE in the isolated vortex core, far from the onset and the boundary layer, is inconsistent with those observed on the wing tip vortices that show steady decay in core TKE with progression. The physical reason behind the experimental result is still unclear. URANS results showed low TKE in the vortex core and high values in the outer regions, which contradicts the EFD data, whereas DES captures a correct trend (Fig. 12). The prediction raises the question: what is the source of high TKE during far-wake progression? Is it maintained by the velocity fluctuation on the free-surface due to the impact of periodic breaking waves originating from the sonar dome? Or it is possible that TKE is convected along the core without any dissipation? Additional experiments and computations are required to investigate these issues.

Overall, the grids used for the static drift case are modest in size compared to those used for the straight-ahead case. The grid studies from all the solvers show that the URANS results improve with grid refinement. Only CFDShip-Iowa provides a systematic grid study using both modest and adaptively refined fine grids. The URANS results do not show significant improvement when grid is refined from $48 \mathrm{M}$ to $84 \mathrm{M}$. Even on the finest grid, URANS predicts excessive decay of the vortices soon after the separation, resulting in poor predictions compared to the data. In addition, URANS grossly underpredicts TKE levels in the vortex core, which is in contradiction to the data. Thus, the errors in URANS predictions are more likely due to modeling issues rather than grid resolution. DES results improve and the resolved TKE levels increase with grid refinement. DES results on the finest $84 \mathrm{M}$ grid provide $>95 \%$ resolved turbulence levels, and predictions are significantly better than URANS. However, DES overpredicts and underpredicts vortex strengths at onset and during progression, respectively. Considering the large grid uncertainties in the simulations, it is difficult to delineate the respective roles played by the discretization and modeling errors.

\section{Unsteady Analysis and Turbulent Kinetic Energy Budgets for $\beta=20$ deg}

6.1 Instability Analysis. As summarized in Table 5, $X$ and $N$ time history FFT shows dominant amplitudes at $f L / U=10$ and 20, 

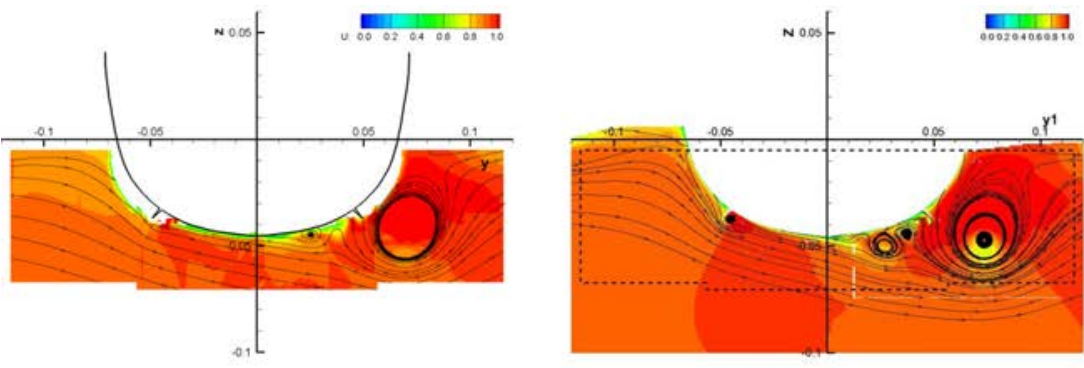

(a)
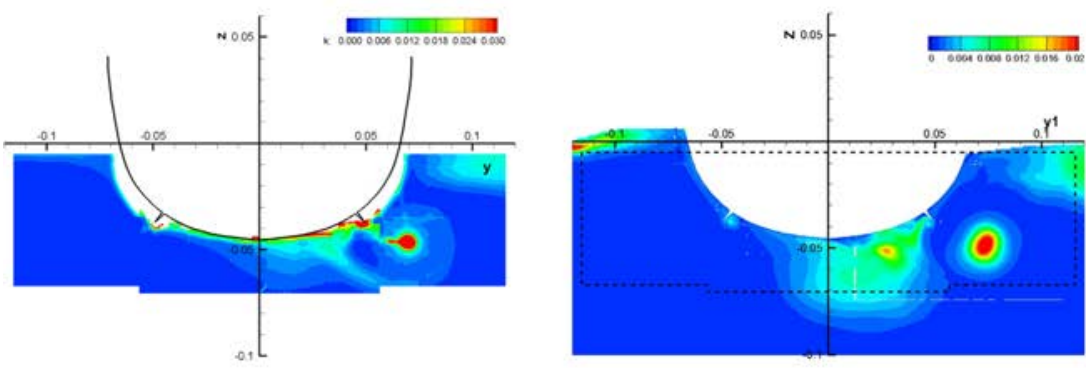

(b)
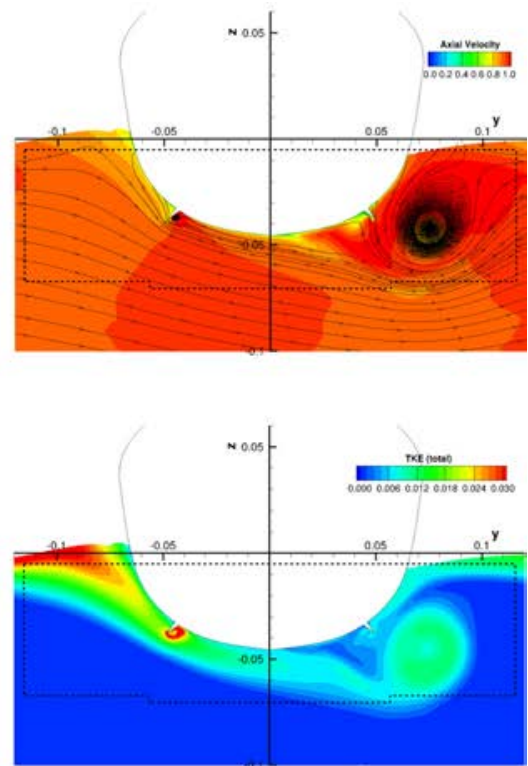

Fig. 12 Contours of (a) axial velocity and crossflow streamlines, and (b) TKE at $x=0.4$ for $\beta=20$ deg. Subplots show: experimental data (left), S2 (middle), and S6 (right).

and those for $Y$ shows at $f L / U=12$ and 25 . For both, the lower frequency shows $15 \%$ higher amplitude than the higher frequency. $X, Y$, and $N$ data show dominant $f L / U=10$ and 20 with latter showing 30-50\% higher amplitude than the former. The same dominant frequencies were also observed for $\beta=0 \mathrm{deg}$ and $10 \mathrm{deg}$ and for the carriage/mount without the model installed. The amplitude of the unsteadiness is significantly large for $X$ (around $10 \% \mathrm{X}$ ) for both $f L / U$. However, the amplitudes for $Y$ and $N$ are comparable to those of CFD, i.e., around 1-2\%. Considering both the CFD and data, it is expected that unsteadiness around $f L /$ $U=10$ and 20 could be partly due to hydrodynamics.

Table 5 summarizes the unsteady analysis conducted using S2. The analysis identified instabilities associated with BSB, SDTV, and BKTV vortex breakdown, LW-FBKV and breaking waves. As shown in Fig. 8(b), the largest RMS pressure was found on the LW bow. Thus, instabilities on the LW bow could be the source of unsteady forces and moment. To further investigate, surface and field point pressure FFT analysis was performed at nine points on the LW bow and four field points, as labeled in Figs. 8(a) and $8(b)$ and listed in Table 5. The BSB separation showed shear layer instability with $f L / U \sim 19$, flapping with $f L / U \sim 14$ and Karman instabilities with $f L / U \sim 5.5$. The surface pressure fluctuation at SDTV separation showed $f L / U \sim 11$. The LW-FBKV showed shear layer instability near its onset. The scaled frequencies are similar to values for canonical and previous surface-piercing NACA24 and KVLCC2 studies [34]. Based on the dominant frequency signatures, the unsteadiness in forces and moment is identified to be due to BSB and SDTV separation.

Figure 9 shows that as SDTV and BKTV progress downstream, the axial velocity and vorticity decrease, pressure increases, and TKE remains nearly constant. The swirl ratio $S_{R}=U_{\theta} / U$, where $U_{\theta}$ is the tangential velocity, showing high values of $0.76-0.82$ at the vortex onset. FFT analysis of seven field point pressures in the vortex cores (shown in Figs. 13(a) and 13(b)) shows that the dominant frequency $S t=f L / U_{0}$ along the vortex core decreases with the progression (Fig. 13(c)). The scaling of the dominant frequency using the distance from the origin of the spiral streamline location $D_{s}$ shows $S t_{X}=f D_{s} / U_{0} \sim 0.75$ to 1.35 , as shown in Fig. $13(d)$.

Previous KVLCC 2 study at $\beta=30$ deg shows open-type crossflow separation from the hull for fore-body side (FSV), after-body side (ASV), and after-body (ABV) vortices. The vortices separated as a circular type, underwent helical mode instability, and transformed into spiral vortices. The transformation of circular to spiral vortices is because of vortex breakdown. As ASV and $\mathrm{ABV}$ progressed downstream, the vortex core axial velocity and vorticity decreased and pressure and TKE increased. FSV also showed local decrease in axial velocity, and increase in TKE and pressure close to vortex breakdown. All the vortices show swirl ratio $S_{R} \sim 0.5$ at vortex breakdown. As shown in Figs. 13(c) and $13(d), S t$ decreases with the progression of the vortices, and $S t_{X}$ is almost constant throughout progression, where averaged $S t_{X} \sim 1.3$ and $\sim 2$ for FSV/ASV and ABV, respectively.

Spiral vortices have been reported for swirling jets [35], wing tip vortices [36], and delta wing leading edge vortices [37]. In these cases, circular vortices exhibit vortex breakdown followed by helical mode instability and transformation into spiral vortices. The breakdown results in a sudden expansion of the vortex core and sharp gradients in vortex core variables, i.e., drop in axial velocity and vorticity, and increase in pressure and TKE [36]. The initiation of the vortex breakdown occurs if the swirl ratio is higher than the critical value $S_{R, c}$. The critical value depends on the flow type, Re, inflow condition, swirl angle with respect to jet axis, etc. [38]. Spall et al. [39] showed that $S_{R, c}$ decreases with Re, i.e., $S_{R, c}=2$ to 3 for $\operatorname{Re}<100$, and 1.6 for large $\operatorname{Re}>10^{4}$ for wing tip vortices. For delta wings, it has been reported that $S_{R, c} \sim 1$ for a range of $\mathrm{Re}$ and wing configurations [38]. In addition, the helical mode instability frequency is inversely proportional to the distance from the breakdown location. Thus, $S t_{X}$ is constant along the core. Gursul [40] reported that $S t_{X}$ ranges from 0.75 to 1.35 for large sweepback wings at different flow conditions.

The vortices for $5415 \beta=20 \mathrm{deg}$ and KVLCC $2 \beta=30 \mathrm{deg}$ have similarities with the swirling jet or delta wing vortices as they show helical mode instability, spiral streamlines/vortices, high swirl at instability inception, and $S t_{X}$ range is consistent with those of delta wings. However, the swirl ratio is lower and large differences are observed in the transition process from circular to spiral vortices, i.e., at vortex breakdown. For swirling jet or delta wing vortices, the transition process is abrupt with large change in vortex size and core variable gradients. For ship flows, the onset/transition process is abrupt but without large change in vortex size and core variables gradients. Nonetheless, we retain the terminology vortex breakdown to describe the transition process. The differences between the ship and aero flows are likely due to global 


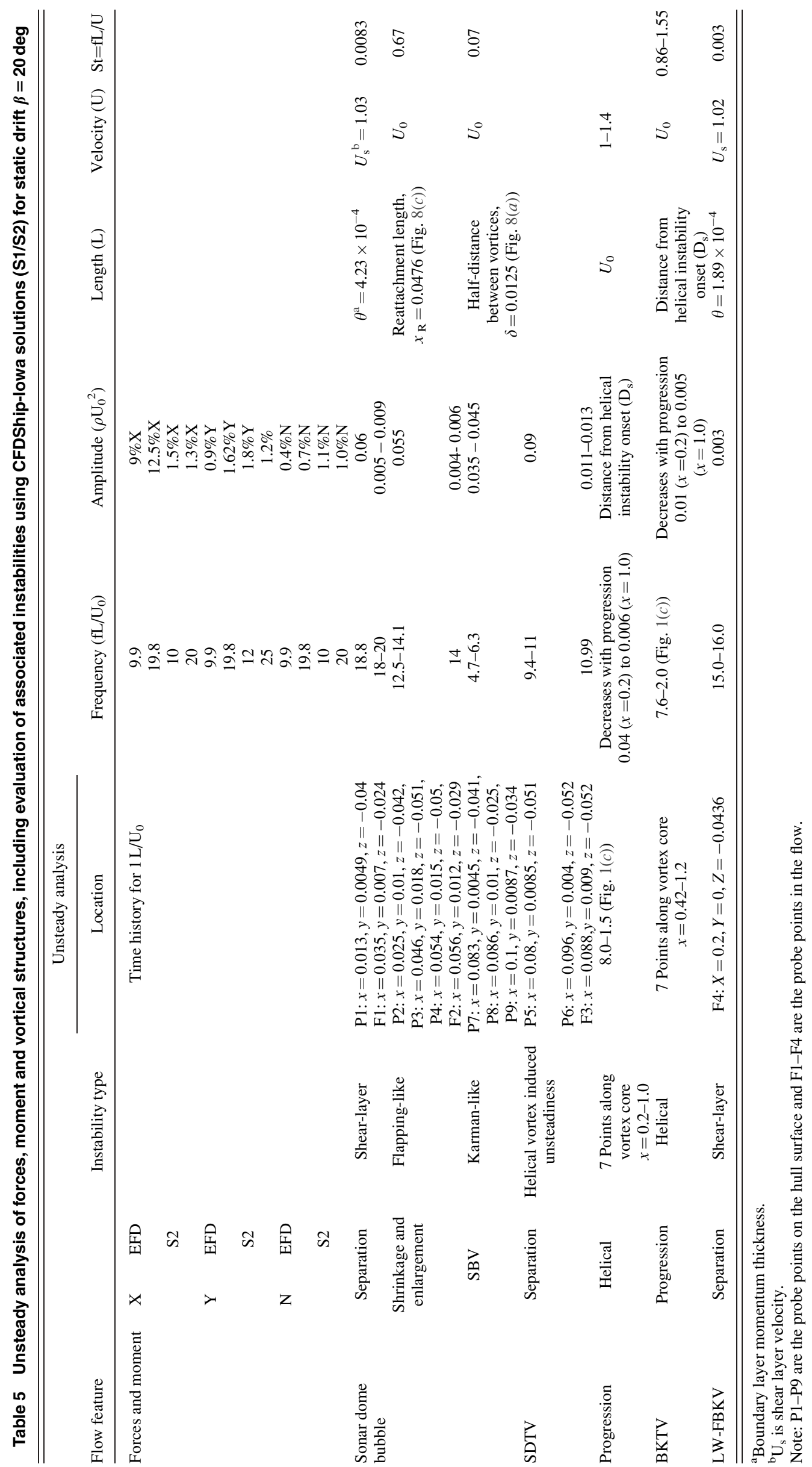




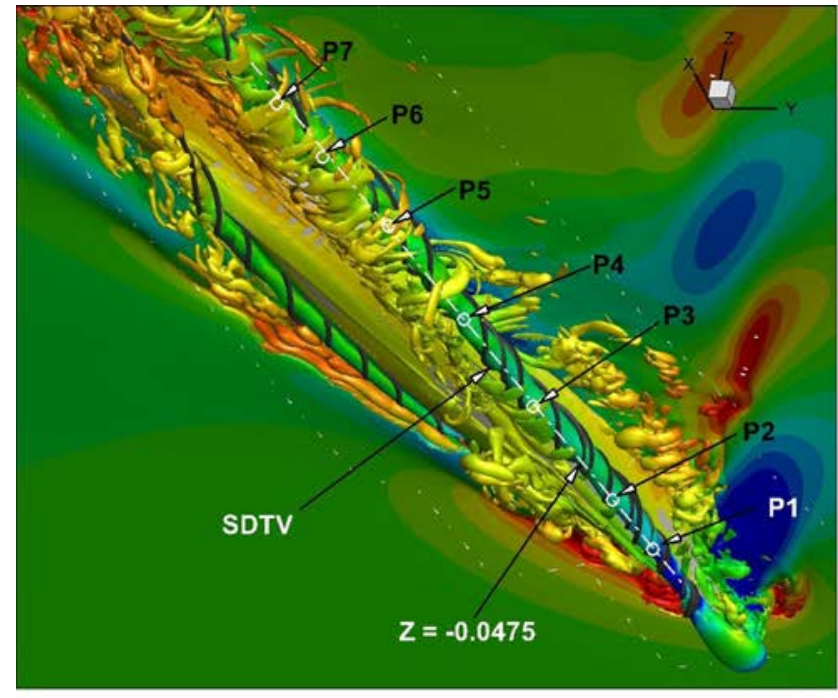

(a)

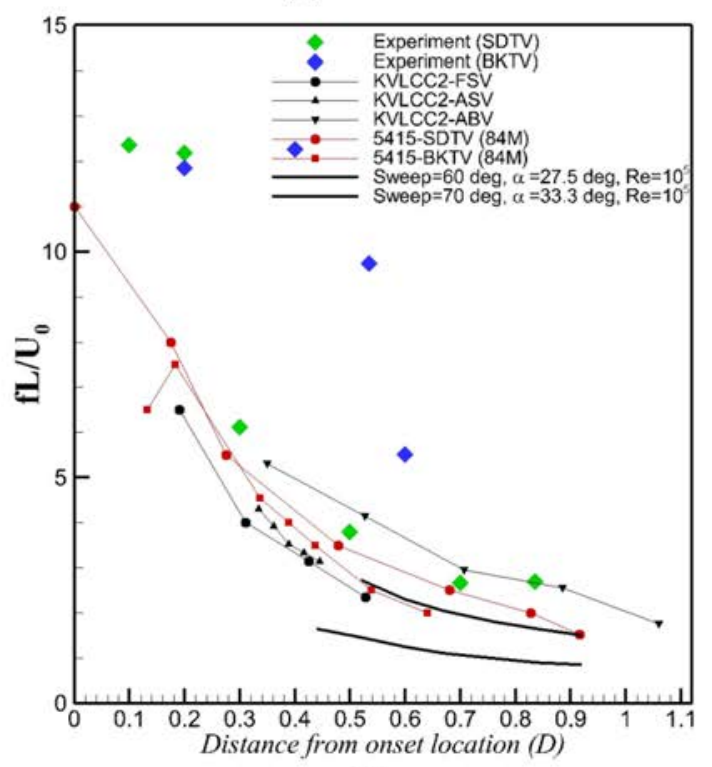

(c)

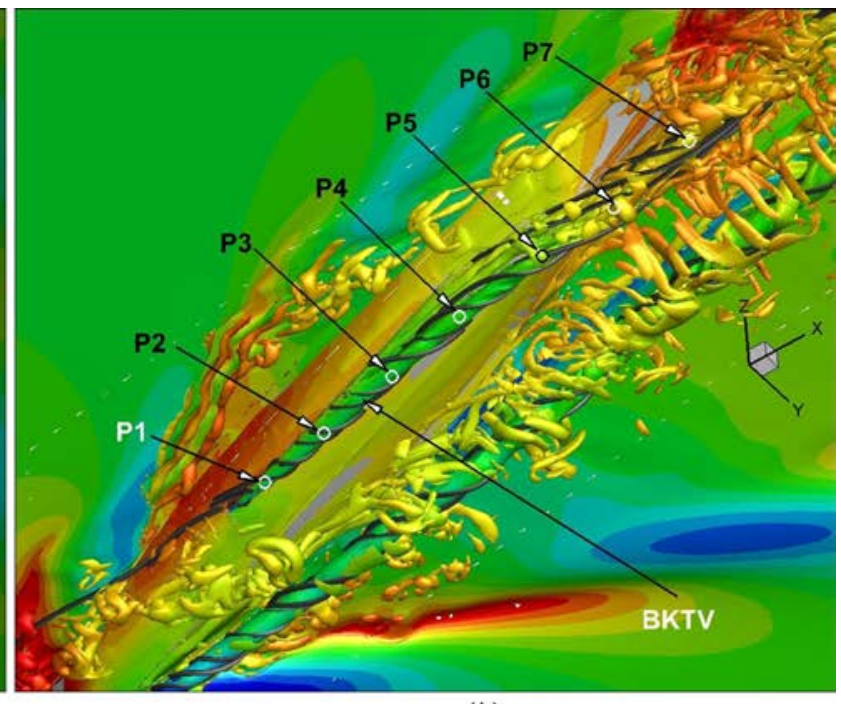

(b)

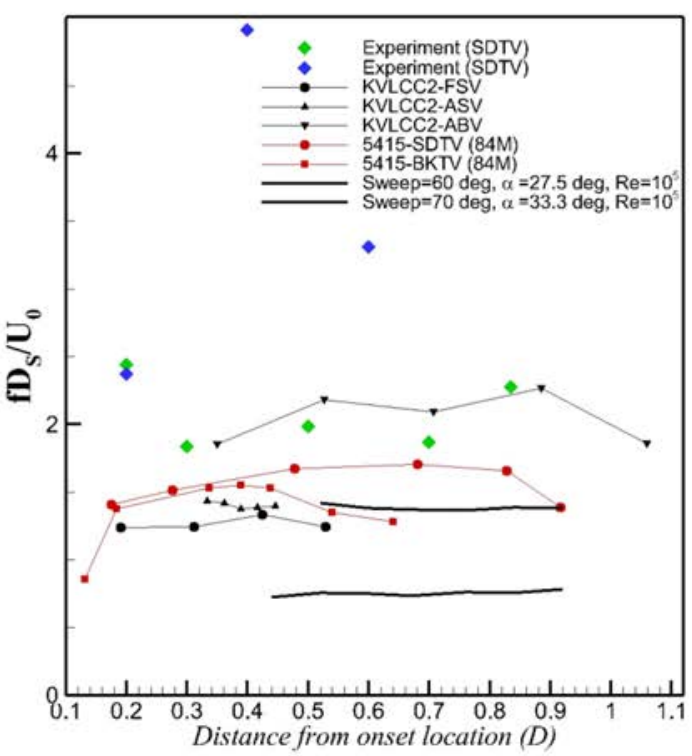

(d)

Fig. 13 Unsteady analysis locations for (a) SDTV and (b) BKTV. Variation of (c) dimensionless frequency and (d) product of frequency and distance from helical instability onset $\left(D_{S}\right)$ for SDTV and BKTV. The predictions are compared with KVLCC2 [4] and delta wing $[40]$ results.

geometry and pressure gradients, and/or the interaction of the ship vortices with the boundary layer and other vortices.

6.2 Turbulent Kinetic Energy Budgets. Figures 14 and 15 summarize the TKE budgets conducted using S2 for BSB and SDTV, respectively. The plots show active LES/URANS regions, modeled and resolved TKE levels, and budgets terms: production $(P r)$, dissipation (modeled) $(\varepsilon)$, convection $(C)$, and turbulent $(T)$, and pressure transport $(P T)$. Diffusion was negligible compared to other terms and is not shown. LES is active for both the separation bubble and vortex advection regions, and URANS is active only in the thin boundary layer. The resolved TKE levels are one to two orders of magnitude larger than modeled TKE.

For $\mathrm{BSB}, \operatorname{Pr}$ is the main producing term and $\varepsilon$ is the main consuming term. Close to the separation, $\mathrm{Pr}$ is the primary TKE source, $\varepsilon$ consumes almost $25 \%$ of TKE, $C$ transports $50 \%$ of TKE along the shear-line, and $T$ and $P T$ transport the rest inside the bubble. Overall, the role budget terms play for the TKE budget is similar with those of separated boundary layer, backward facing step recirculation, and LW separation bubble for KVLCC2 $\beta=30 \mathrm{deg}$ flow [4]. Away from the separation as the bubble radius increases, $\operatorname{Pr}$ decreases but is still significant, dissipation is negligible, and $T$ and $P T$ are the primary sinks that transport TKE inside the bubble. The transport of TKE inside the separation bubble is similar to free surface-induced separation bubble for 5415 [41] and KVLCC2 separation bubble. However, both the previous studies showed $C$ as the primary transport term. Close to the attachment, $P r$ is the primary TKE source, but is almost an order of magnitude smaller than that close to the separation. $P T$ is the primary sink, which transports TKE inside the bubble.

For SDTV, TKE peaks close to the breakdown and decays with progression. $\operatorname{Pr}$ and $\varepsilon$ are the primary source and sink terms, respectively. Peak $P r$ occurs close to the vortex breakdown, and decreases with progression. $\varepsilon$ is aligned with the vortex core, increases with progression, and $\left|\varepsilon_{P} / P r_{p}\right| \sim 2$, where the subscript $p$ denotes the peak value. $C$ transports TKE along vortex core and $\left|C_{p} / P r_{p}\right| \sim 0.5$. Both $T$ and $P T$ transport energy away from vortex core such that $\left|T_{p} / P r_{p}\right| \sim 1.25$ and $\left|P T_{p} / P r_{p}\right| \sim 0.7$. The TKE distribution in the core, peak $\mathrm{Pr}$ at the breakdown, and the role of $C$ 


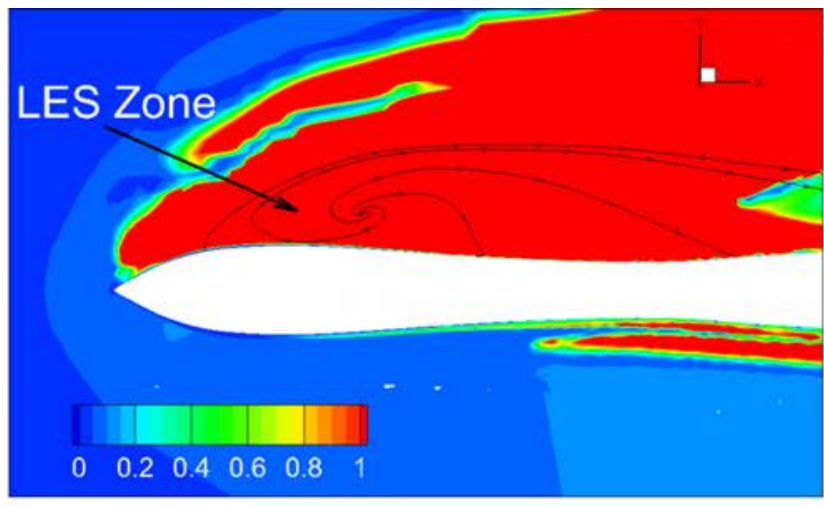

(a)

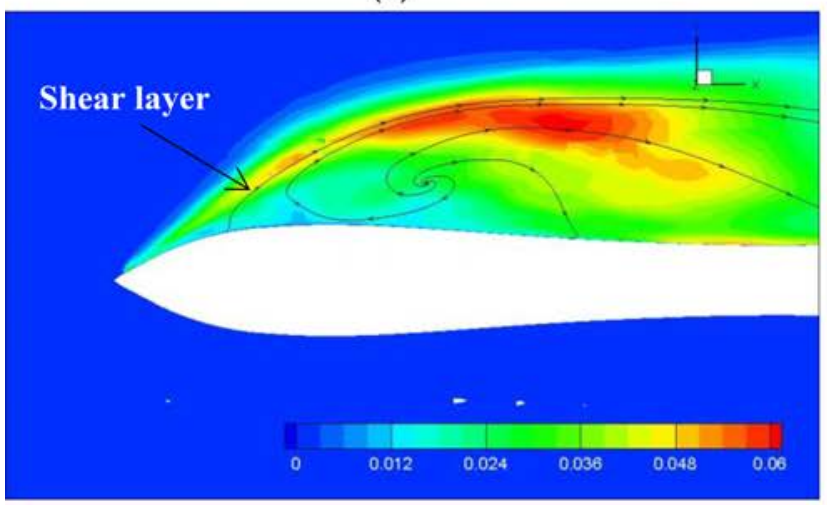

(c)

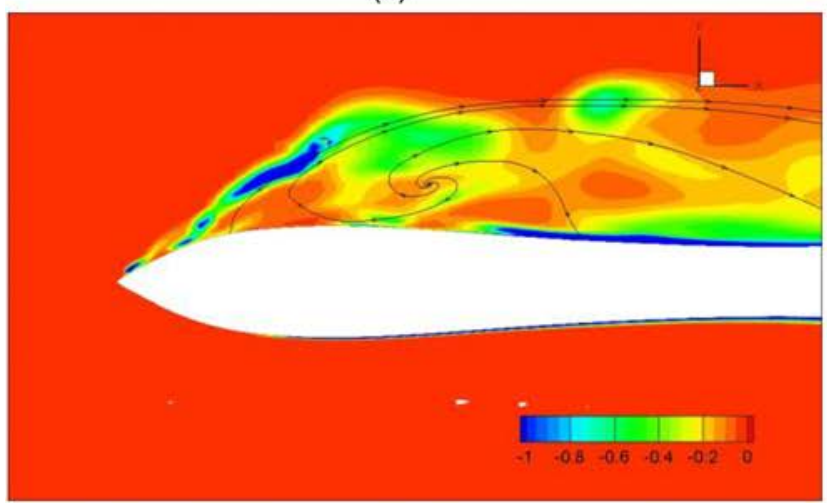

(e)

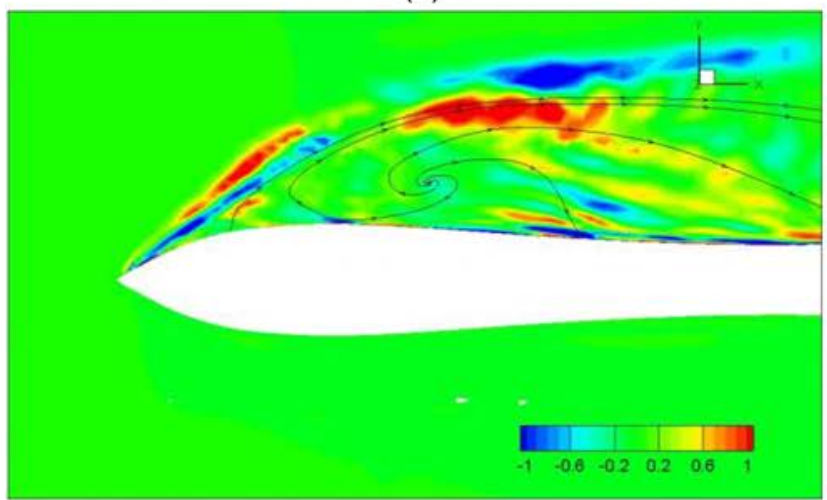

(g)

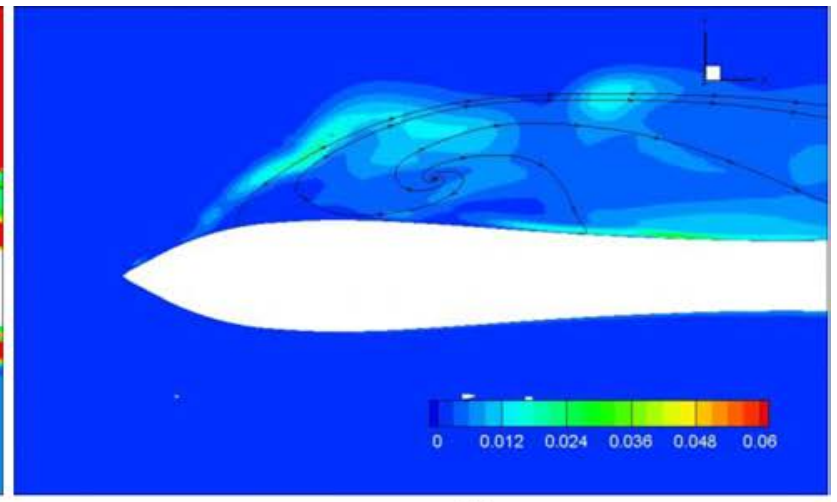

(b)

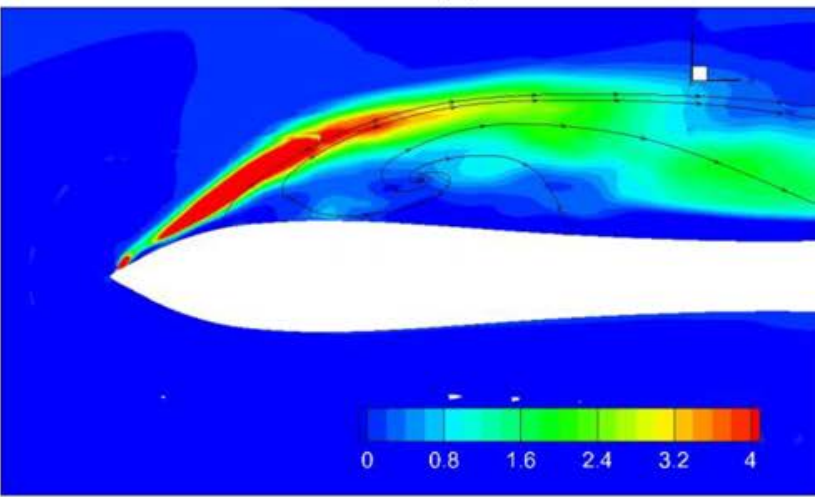

(d)

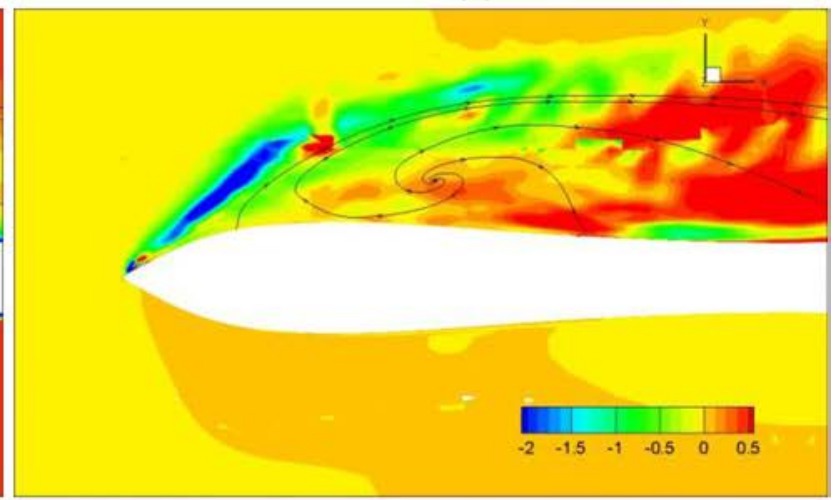

(f)

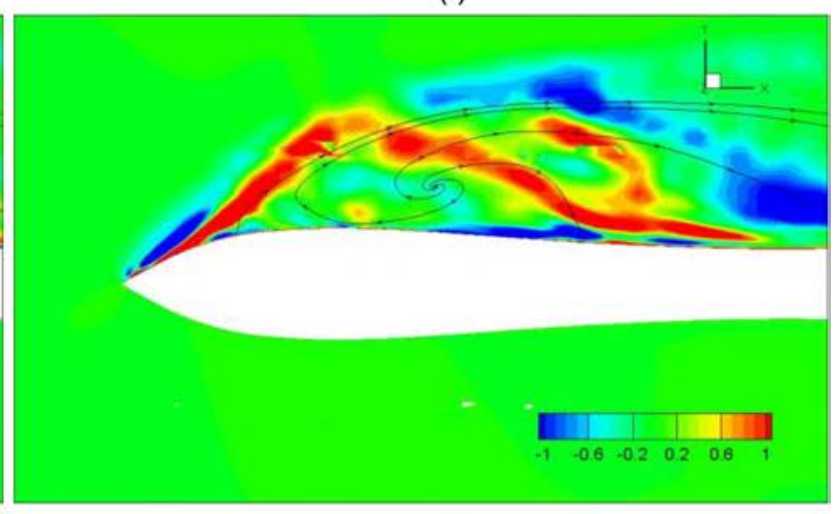

(h)

Fig. 14 Contours of TKE budget terms in the LW-SDB on $z=-0.04$ plane, marked in Fig. 8(a). Contour plots show: (a) URANS/LES regions, where regions flooded in red are LES zone and those in blue are URANS zone, (b) modeled and (c) resolved TKE. TKE budget terms ( $d)$ production, $(e)$ dissipation, $(f)$ convection, $(g)$ turbulent transport, and $(h)$ pressure transport. The black lines show the mean flow streamline. 


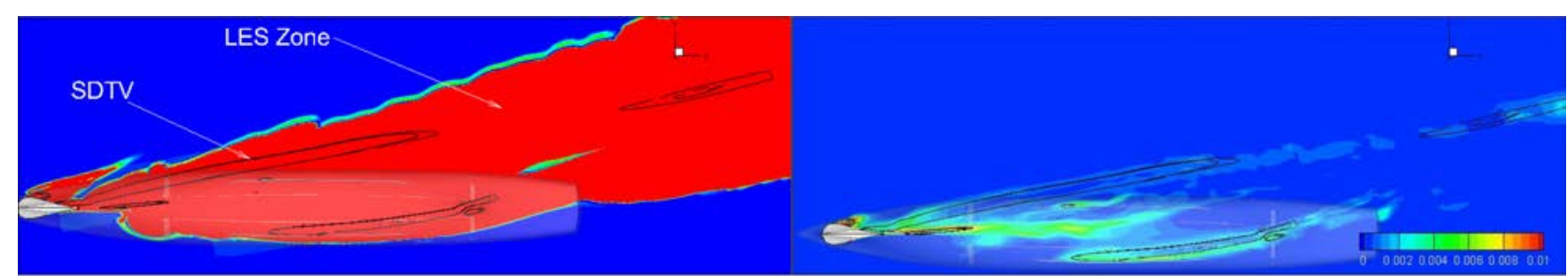

(a)

(b)

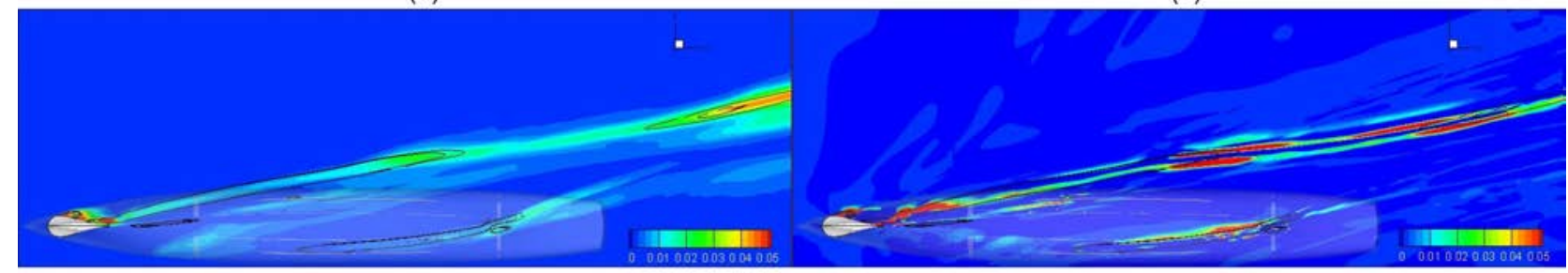

(c)

(d)

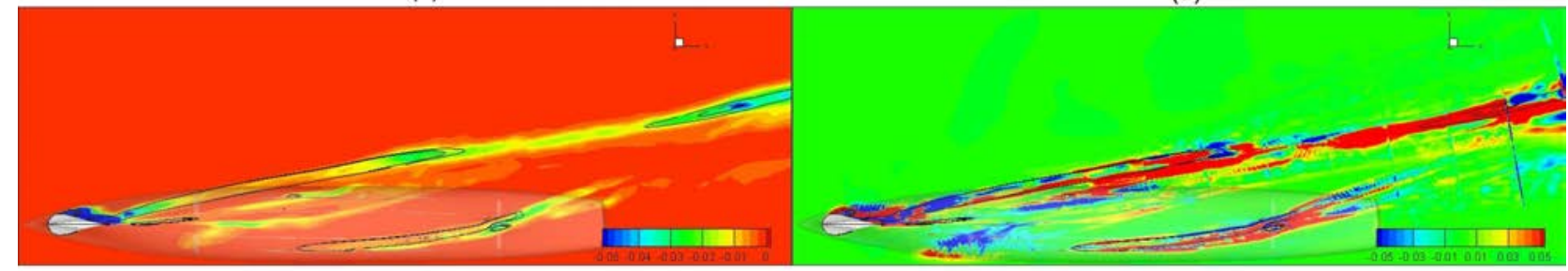

(e)

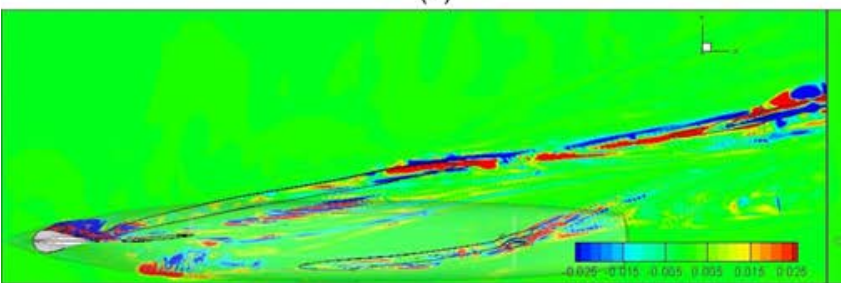

(g) (f)

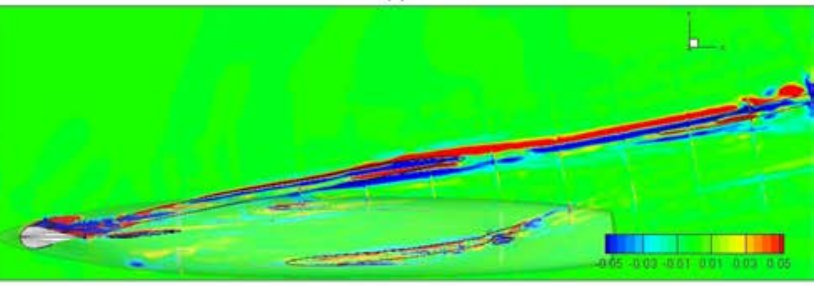

(h)

Fig. 15 TKE budget along SDTV core on $z=-0.0475$ plane, marked in Fig. 13(a). Contour plots show: (a) URANS/LES regions, where regions flooded in red are LES zone and those in blue are URANS zone, $(b)$ modeled and $(c)$ resolved TKE. TKE budget terms: $(d)$ production, $(e)$ dissipation, $(f)$ convection, $(g)$ turbulent transport, and $(h)$ pressure transport. The black lines show the $Q=500$ and 1000 contours.

and $T$ in TKE distribution are consistent with those observed in delta wing [42] and KVLCC2 flows. However, both the 5415 and KVLCC2 flows show high dissipation compared to the delta wing flow. For 5415, the peak $C$ occurs during early progression, whereas for KVLCC2, they occur during late progression. Further, $P T$ transports energy away from the core and along the core for 5415 and KVLCC2, respectively.

\section{Conclusions and Future Work}

Integrated TPIV experiments and CFD prediction provide credible description of the onset and progression of 3D vortex separation for 5415 at both straight-ahead $\beta=0 \mathrm{deg}$ and static drift $\beta=20 \mathrm{deg}$, including assessment of CFD solutions for forces and moment, wave elevation, and mean and turbulent flows in the vortex core.

In the experiments, $X$ and $Y / N$ show quadratic and cubic increase with $\beta$, as expected. Both $\beta$ show similar errors $\overline{|E|}=3.4 \% D$ and uncertainties for forces and moment, and all of the solutions are validated at $U_{V i}=4 \% D$ interval and $\mathrm{N}$-version validated at $U_{S O A i}=7 \% D$ interval. The mean code solution is validated at $U_{S o A}=5 \% D$ interval. The largest to smallest uncertainties are due to solution scatter, experimental, and numerical. The trends for $\beta=0$ deg errors are similar to those for G2010 5415, and errors and uncertainties for T2015 KCS test cases. The errors for $\beta=20 \mathrm{deg}$ are substantially improved compared to SIMMAN 2008, which is attributed to the use of finer grids. Thus, CFD predictive capability for forces and moment is comparable for both straight-ahead and static drift conditions even for large drift angles.

The $\beta=20 \mathrm{deg}$ wave elevations are asymmetric and have two times higher amplitude compared to $\beta=0 \mathrm{deg}$, but the total wave envelop angle $\sim 37 \mathrm{deg}$ remains same. The average errors and uncertainties for the wave elevation are $|E|=7 \% D$. For both $\beta$, most solutions are validated at $U_{V i}=7 \% D$ and $\mathrm{N}$-version validated at $U_{S O A}=8 \% D$. The mean code is validated at $U_{S O A}=7 \% D$. CFD predictive capability is somewhat reduced for static drift case compared to the straight ahead case likely due to deficiencies in the prediction of breaking waves. The experimental, numerical, and solution scatter uncertainties show equal contribution. Future CFD workshops should include test case for model ship with breaking waves for more rigorous assessment of CFD capability.

The forces and moment and wave elevation predictions do not show significant dependence on turbulence modeling, or wall modeling as long as near-wall grid resolution $y^{+} \sim 30$, or freesurface modeling. Results improve with grid resolution, but do not show significant improvements for grids $>50 \mathrm{M}$ cells. Wall blockage does not affect the force predictions for $\beta=0 \mathrm{deg}$, but its effect for $\beta=20 \mathrm{deg}$ forces and moment needs further investigation. Free-surface modeling shows difference in the wave breaking pattern for $\beta=20 \mathrm{deg}$, where VoF predicts more active breaking than level-set.

The primary vortices for $\beta=0$ deg are SDV and FBKV originating from crossflow separation on the side and trailing edge of the sonar dome, respectively. Secondary BKV originates from the 
bilge keel tip. The flow is symmetric with respect to the center plane and the vortices show clockwise rotation on the SS (when looking forward). The $\beta=20 \mathrm{deg}$ flow is asymmetric with respect to the center plane, and the crossflow drift induces vortex progression. The primary vortices are counter clockwise rotating SDTV and BKTV originating from the LW sonar dome side and WW bilge keel, respectively. CFD predictions also show several secondary vortices on the LW side and wave breaking, which could not be confirmed from the experiments. The characteristics of SDTV and BKTV are different from SDV and FBKV. In particular, the former progresses away from the hull, whereas the latter progresses close to the hull and interacts with the boundary layer. SDTV and BKTV show a sharp jump in core flow downstream of onset, which are consistent with vortex breakdown and helical mode instability. For the $\beta=20$ deg vortices, the velocity deficit increases with progression, whereas it recovers for $\beta=0 \mathrm{deg}$. They show one to two orders of magnitude higher vortex strength and TKE compared to the $\beta=0$ deg vortices, and different decay rates.

The CFD vortex onset and overall structure predictions for both $\beta$ show similar errors and scatter. The errors $|E| /$ scatter $\sigma_{S}$ for the vortex location $(4 \% D / 3 \% \bar{S})$ and axial velocity $(8 \% D / 6 \% \bar{S})$ are relatively small, but are large for $Q(143 \% D / 117 \% \bar{S}), \omega_{x}$, and TKE $(60 \% D / 40 \% \bar{S})$. The intervals of solution and $\mathrm{N}$-version validation are large: $U_{V i}=20 \% \mathrm{D}, U_{S O A}=70 \% D$, and $U_{S O A}=40 \% D$. Most $R$ and $U$ predictions are solution and $\mathrm{N}$-version validated at $12 \%$ interval, so is the mean code prediction. Whereas, only a few predictions for $Q, \omega_{x}$, and TKE are solution and N-version validated, and the mean code is not. Both $R$ and $U$ show similar experimental, numerical, solution scatter uncertainties. For $Q, \omega_{x}$, and TKE, solution scatter uncertainties are five times larger than the numerical and experimental uncertainties

Unsteady analysis shows that the $\beta=20 \mathrm{deg}$ bow separation bubble exhibits shear layer, Karman, and flapping instabilities with scaling laws typical of canonical flows and previous ship studies. Spilling and plunging wave breaking are predicted on WW and LW bow, respectively. SDTV and BKTV exhibit vortex breakdown and helical mode instability with scaling laws similar to those for delta wings. The shear layer separations associated with bow bubble and SDTV induce fluctuation in the hull pressure distribution resulting in unsteady forces and moment. TKE budgets for bow separation bubble and SDTV have similarities with those for canonical flows and previous ship studies for the TKE distribution, production, and transport mechanisms. However, it shows some differences in the role of the convection, turbulent transport, and pressure transport terms, especially the latter is more dominant compared to canonical flows.

Overall, CFD predictive capability for 3D vortex separation is comparable for both straight ahead and static drift even for large drift angles, i.e., the computations predict onset and overall vortex structures well, but with large errors and uncertainties for vortex progression. Confirmation of the relative strengths of SDV and FBKV and their interaction (along with those of $\mathrm{BKV}$ ) on the mid-/after-body resulting in the nominal wake remain an outstanding issues for $\beta=0 \mathrm{deg}$. Experiments show high TKE level in the SDTV core throughout progression. Further investigation is required to understand the physical mechanism behind the sustained TKE level. Improvement in $\beta=0 \mathrm{deg}$ solutions require improvement in turbulence models, whereas for $\beta=20 \mathrm{deg}$, both better grids and turbulence models are required. For improved grid design, solution-adaptive mesh refinement offers a cost-effective meshing strategy. However, the regularized Hessian of the pressure-based refinement fails to refine the grid all along the vortex. The overset methodology or region-based adaptation is a viable alternative for solution-based grid refinement, but is more time consuming and requires user input. Full Reynolds stress transport model could improve URANS predictions; however, URANS is not well suited for unsteady flows with dominant coherent vortical structures. Advanced hybrid RANS/LES models are required to address the
DES modeling issues, including explicit LES models and physics-based RANS-LES transition.

\section{Acknowledgment}

The U.S. Office of Naval Research under Grant N00014-10-10017 sponsored the research at the University of Iowa under the administration of Drs. Thomas Fu, Woei-Min Lin, and Ki-Han Kim. Drs. Patrick Purtell and Ki-Han Kim provided guidance to the international collaboration over the course of this study and Dr. Kim graciously aided in editing. Dr. Stephan Hitzel, Airbus, provided consultation on the assessment of the ship-flow vortex breakdown. The contribution of MARIN to this research is partially funded by the Dutch Ministry of Economic Affairs.

\section{Nomenclature}

$$
\begin{aligned}
& E_{i} \% D=\left(\left(D-S_{i}\right) / D\right) \times 100=\text { comparison error, where } D \\
& \left\langle E_{i}\right\rangle \% D=(1 / P) \sum_{p=1} \\
& P\left|D_{p}-S_{p i} / D_{p}\right| \times 100=\text { Averaged comparison error } \\
& \bar{E}=\frac{1}{N_{s}} \sum_{i=1}^{N_{s}} E_{i}=\text { averaged error } \\
& \overline{|E|}=\frac{1}{N_{s}} \sum_{i=1}^{N_{s}}\left|E_{i}\right| \geq|\bar{E}|=\text { averaged absolute error } \\
& H=\boldsymbol{U} \cdot \boldsymbol{\omega} /|\boldsymbol{U}||\boldsymbol{\omega}|=\text { helicity, where } \boldsymbol{U} \text { and } \boldsymbol{\omega} \text { are } \\
& N_{s}=\text { number of solutions } \\
& P_{S i}=2 \sigma_{S}=\mathrm{N} \text {-version verification pre- } \\
& P_{\bar{S}}=2 \sigma_{S} / \sqrt{N_{s}}=\mathrm{N} \text {-version validation preci- } \\
& P_{\left|E_{i}\right|}=2 \sigma_{|E|}=\mathrm{N} \text {-version validation preci- } \\
& P \overline{|E|}=2 \sigma_{|E|} / \sqrt{N_{s}}=\mathrm{N} \text {-version validation preci- } \\
& Q=(1 / 2)\left[\left\|\mathbf{\Omega}^{2}\right\|-\left\|\mathbf{S}^{2}\right\|\right]=\text { second invariant of rate-of- } \\
& U, V, W=\text { mean velocities along } x, y \\
& \text { and } z \text { directions, } \\
& \text { respectively } \\
& U_{D}=\text { Experimental uncertainty } \\
& U_{0}=\text { freestream velocity } \\
& U_{I i}, U_{G i}, U_{T i}=\text { iterative, grid and time step } \\
& \text { uncertainties in CFD, } \\
& U_{S N i}=\sqrt{U_{I i}^{2}+U_{G i}^{2}+U_{T i}^{2}} \% S_{i}=\text { numerical uncertainty in } \\
& \text { CFD solution } \\
& U_{\overline{S N}}=\frac{1}{N_{s}} \sum_{i=1}^{N_{s}} U_{S N i}=\text { averaged numerical }
\end{aligned}
$$


$x, y, z=$ coordinates along streamwise, spanwise and freesurface normal directions, respectively

$X, Y, N=$ longitudinal force, side force and yaw moment, respectively

$\zeta=$ wave-elevation

$\sigma_{S}=\sqrt{\left(1 / N_{s}-1\right) \sum_{i=1}^{N_{s}}\left(S_{i}-\bar{S}\right)^{2}}$

$=\sqrt{\left(1 / N_{s}-1\right) \sum_{i=1}^{N_{s}}\left(E_{i}-\bar{E}\right)^{2}}=\sigma_{E}=\begin{aligned} & \text { standard deviation in } \\ & \text { solutions }\end{aligned}$

$$
\sigma_{|E|}=\sqrt{\frac{1}{N_{s}-1} \sum_{i=1}^{N_{s}}\left(\left|E_{i}\right|-\overline{|E|}\right)^{2}}=\begin{aligned}
& \text { standard deviation of abso- } \\
& \text { lute error }
\end{aligned}
$$
$\omega_{x}, \omega_{y}, \omega_{z}=$ vorticity components along $x, y$ and $z$ directions, respectively

\section{References}

[1] Gorski, J. J., Miller, R. W., and Coleman, R. M., 2004, "The Use of a RANS Code in the Design and Analysis of a Naval Combatant," 25th Symposium on Naval Hydrodynamics, St. John's, NF, Canada, Aug. 8-13, pp. 144-159.

[2] Larsson, L., Stern, F., and Visonneau, M., 2014, Numerical Ship Hydrodynamics: An Assessment of the Gothenburg 2010 Workshop, Springer, The Netherlands, p. 318.

[3] Stern, F., Agdrup, K., Kim, S. Y., Hochbaum, A. C., Rhee, K. P., Quadvlieg, F. Perdon, P., Hino, T., Broglia, R., and Gorski, J., 2011, "Experience From SIMMAN 2008: The First Workshop on Verification and Validation of Ship Maneuvering Simulation Methods," J. Ship Res., 55(2), pp. 135-147.

[4] Xing, T., Bhushan, S., and Stern, F., 2012, "Unsteady Vortical Flow and Turbulent Structures for a Tanker Hull Form at Large Drift Angles," Ocean Eng., 55, pp. 23-43.

[5] Visonneau, M., Deng, G. B., Guilmineau, E., Queutey, P., and Wackers, J., 2016, "Local and Global Assessment of the Flow Around the Japan Bulk Carrier With and Without Energy Saving Devices at Model and Full Scale," 31st Symposium on Naval Hydrodynamics, Monterey, CA, Sept. 11-16.

[6] Larsson, L., Stern, F., Visonneau, M., Hino, T., Hirata, N., and Kim, J., 2018 "Tokyo 2015: A Workshop on CFD in Ship Hydrodynamics," Workshop Proceedings, Tokyo, Dec. $2-4$

[7] Stern, F., and Toxopeus, S. L., 2017, "Sea Facet Experimental and Computational Analysis Activities on Onset and Progression of SeparationConclusions and Future Research," North Atlantic Treaty Organization Science \& Technology Organization, Brussels, Belgium, Technical Report No. TRAVT-183.

[8] Yoon, H., Longo, J., Toda, Y and Stern, F, 2015 "Benchmark CFD Validation Data for Surface Combatant 5415 in PMM Maneuvers-Part II: PhaseAveraged Stereoscopic PIV Flow Field Measurements," Ocean Eng., 109(15), pp. 735-750.

[9] Stern, F., Wilson, R. V., Coleman, H. W., and Paterson, E. G., 2001, "Comprehensive Approach to Verification and Validation of CFD Simulations-Part 1: Methodology and Procedures," ASME J. Fluids Eng., 123(4), pp. 793-802.

[10] Xing, T., and Stern, F., 2010, "Factors of Safety for Richardson Extrapolation," ASME J. Fluids Eng., 132(6), p. 061403

[11] Eça, L., and Hoekstra, M., 2014, "A Procedure for the Estimation of the Numerical Uncertainty of CFD Calculations Based on Grid Refinement Studies," J. Comput. Phys., 262, pp. 104-130.

[12] Stern, F., Diez, M., Sadat-Hosseini, H., Yoon, H., and Quadvlieg, F., 2017, "Statistical Approach for Computational Fluid Dynamics State-of-the-Art Assessment: N-Version Verification and Validation," ASME J. Verif., Validation Uncertainty Quantif., 2(3), p. 031004.

[13] Bhushan, S., Yoon, H., Stern, F., Guilmineau, E., Visonneau, M., Toxopeus, S., Simonsen, C., Aram, S., Kim, S. E., and Grigoropoulos, G., 2015, "CFD Validation for Surface Combatant 5415 Straight Ahead and Static Drift 20 Degree Conditions," IIHR-Hydroscience \& Engineering, Iowa City, IA, Report No. 493.

[14] Longo, J., Shao, J., Irvine, M., and Stern, F., 2007, "Phase-Averaged PIV for the Nominal Wake of a Surface Ship in Regular Head Waves," ASME J. Fluid Eng., 129 (5), pp. 524-540.

[15] Bhushan, S., Carrica, P., Yang, J., and Stern, F., 2011, "Scalability and Validation Study for Large Scale Surface Combatant Computations Using CFDShipIowa,” Int. J. High Perform. Comput. Appl., 25(4), pp. 466-487.
[16] Huang, J., Carrica, P. M., and Stern, F., 2008, "Semi-Coupled Air/Water Immersed Boundary Approach for Curvilinear Dynamic Overset Grids With Application to Ship Hydrodynamics," Int. J. Numer. Methods Fluids, 58(6), pp. 591-624.

[17] Vaz, G., Jaouen, F. A. P., and Hoekstra, M., 2009, "Free-Surface Viscous Flow Computations. Validation of URANS Code FRESCO," 28th International Conference on Ocean, Offshore and Arctic Engineering (OMAE), Honolulu, HI May 31-June 5.

[18] Wackers, J., Deng, G. B., Leroyer, A., Queutey, P., and Visonneau, M., 2012, "Adaptive Grid Refinement Algorithm for Hydrodynamic Flows," Comput. Fluids, 55, pp. 80-100.

[19] Guilmineau, E., Deng, G. B., Leroyer, A., Queutey, P., Visonneau, M. and Wackers, J., 2017, "Assessment of Hybrid RANS-LES Formulations for Flow Simulation Around the Ahmed Body," VII European Congress on Computational Methods in Applied Sciences and Engineering, M. Papadrakakis, V. Papadopoulos, G. Stefanou, V. Plevris eds., Crete Island, Greece, June 5-10.

[20] Kim, S. E., Rhee, B. J., and Miller, R. W., 2014, "Anatomy of Turbulent Flow Around DARPA SUBOFF Body in a Turning Maneuver Using High-Fidelity RANS Computations," Int. Shipbuild. Prog., 60(1), pp. 207-231.

[21] Menter, F. R., 1994, "Two-Equation Eddy-Viscosity Turbulence Models for Engineering Applications," AIAA J., 32(8), pp. 1598-1605.

[22] Spalart, P., 2009, "Detached Eddy Simulation," Annu. Rev. Fluid Mech., 41(1), pp. $181-202$.

[23] Menter, F. R., Kuntz, M., and Langtry, R., 2003, “Ten Years of Industrial Experience With the SST Turbulence Model," Turbul. Heat Mass Transfer 4, K. Hanjalic, Y. Nagano, and M. Tummers, eds., Begell House, Inc., pp. 625-632.

[24] Longo, J., and Stern, F., 2002, "Effects of Drift Angle on Model Ship Flow," Exp. Fluids, 32(5), pp. 558-569.

[25] Stern, F., Longo, J., Zhang, Z. J., and Subramani, A. K., 1996, "Detailed BowFlow Data and CFD for a Series $60 \mathrm{C}_{\mathrm{B}}=0.6$ Ship Model for Froude Number 0.316,” J. Ship Res., 40(3), pp. 193-199.

[26] Olivieri, A., Pistani, F., Avanzini, G., Stern, F., and Penna, R., 2001, “Tank Experiments of Resistance, Sinkage and Trim, Boundary Layer, Wake and Free Surface Flow Around a Naval Combatant INSEAN 2340 Model,” IIHR, Report, No. 421

[27] Surana, A., Grunberg, O., and Haller, G., 2006, "Exact Theory of ThreeDimensional Flow Separation-Part I: Steady Separation,” J. Fluid Mech., 564 pp. 57-103.

[28] Simpson, R. L., 1996, "Aspects of Turbulent Boundary Layer Separation," Prog. Aerosp. Sci., 32(5), pp. 457-521.

[29] Delery, J. M., Legendre, R., and Werle, H., 2001, "Toward the Elucidation of Three-Dimensional Separation," Annu. Rev. Fluid Mech., 33(1), pp. $129-154$.

[30] Wilson, R. V., Carrica, P. M., and Stern, F., 2006, "URANS Simulations for a High-Speed Transom Stern Ship With Breaking Waves," Int. J. Comput. Fluid Dyn., 20(2), pp. 105-125.

[31] Zhang, Z. J., and Stern, F., 1996, "Free-Surface Wave-Induced Separation," ASME J. Fluids. Eng., 118(3), pp. 546-554.

[32] Egolf, T. A., Wake, B., and Berezin, C., 2000, "Recent Rotor Wake Simulation and Modeling Studies at United Technologies Corporation," AIAA Paper No. AIAA-2000-0115

[33] Bhushan, S., Xing, T., Visonneau, M., Wackers, J., Deng, G., Stern, F., and Larsson, L., 2014, "Chapter 7: Post Workshop Computations and Analysis for KVLCC2 and 5415," Numerical Ship Hydrodynamics: An Assessment of the Gothenburg 2010 Workshop, L., Larsson, F. Stern, and M. Visonneau, eds. Springer, Dordrecht, The Netherlands.

[34] Bhushan, S., Xing, T., and Stern, F., 2012, "Vortical Structures and Instability Analysis for Athena Wetted Transom Flow With Full-Scale Validation," ASME J. Fluids Eng., 134(3), p. 031201.

[35] Gallaire, F., and Chomaz, J. M., 2003, "Mode Selection in Swirling Jet Experiments: A Linear Stability Analysis," J. Fluid Mech., 494, pp. 223-253.

[36] Nelson, R. C., and Pelletier, A., 2003, "The Unsteady Aerodynamics of Slender Wings and Aircraft Undergoing Large Amplitude Maneuvers," Prog. Aerosp. Sci., 39(2-3), pp. 185-248.

[37] Gursul, I., 2009, "Unsteady Aspects of Leading-Edge Vortices," Report No. NATO-RTO-TR-AVT-080.

[38] Greenwell, D. I., 2009, "Engineering Models of Delta Wing Vortex Breakdown and Its Effect on Aerodynamic Characteristics," Report No. NATO-RTO-TRAVT-080.

[39] Spall, R. E., Gatski, T. B., and Grosch, C. E., 1987, “A Criterion for Vortex Breakdown," Phys. Fluids, 30(11), pp. 3434-3440.

[40] Gursul, I., 1994, "Unsteady Flow Phenomena Over Delta Wings at High Angle of Attack," AIAA J., 32(2), pp. 225-231.

[41] Xing, T., Kandasamy, M., and Stern, F., 2007, "Unsteady Free-Surface Wave-Induced Separation: Analysis of Turbulent Structures Using Detached Eddy Simulation and Single-Phase Level Set," J. Turbul., 8(44), pp. $1-35$

[42] Panagiotou, P., Sideridis, A., Yakinthos, K., and Goula, A., 2015, “Turbulence Kinetic Energy Balance in the Wake of a Sharp-Edged Highly Swept Delta Wing," Flow, Turbul. Combust., 25(1), pp. 121-142. 